\title{
Breakup reaction models for two- and three-cluster projectiles
}

\author{
D. Baye and P. Capel
}

\begin{abstract}
Breakup reactions are one of the main tools for the study of exotic nuclei, and in particular of their continuum. In order to get valuable information from measurements, a precise reaction model coupled to a fair description of the projectile is needed. We assume that the projectile initially possesses a cluster structure, which is revealed by the dissociation process. This structure is described by a few-body Hamiltonian involving effective forces between the clusters. Within this assumption, we review various reaction models. In semiclassical models, the projectile-target relative motion is described by a classical trajectory and the reaction properties are deduced by solving a time-dependent Schrödinger equation. We then describe the principle and variants of the eikonal approximation: the dynamical eikonal approximation, the standard eikonal approximation, and a corrected version avoiding Coulomb divergence. Finally, we present the continuum-discretized coupled-channel method (CDCC), in which the Schrödinger equation is solved with the projectile continuum approximated by square-integrable states. These models are first illustrated by applications to two-cluster projectiles for studies of nuclei far from stability and of reactions useful in astrophysics. Recent extensions to threecluster projectiles, like two-neutron halo nuclei, are then presented and discussed. We end this review with some views of the future in breakup-reaction theory.
\end{abstract}

\footnotetext{
D. Baye

Physique Quantique, C.P. 165/82 and Physique Nucléaire Théorique et Physique Mathématique, C.P. 229, Université Libre de Bruxelles, B 1050 Brussels, Belgium, e-mail: dbaye@ulb.ac.be

P. Capel

National Superconducting Cyclotron Laboratory, Michigan State University, East Lansing MI48824, USA, e-mail: capelenscl.msu.edu
} 


\section{Introduction}

The advent of radioactive ion beams has opened a new era in nuclear physics by providing the possibility to study nuclei far from stability. In particular the availability of these beams favoured the discovery of halo nuclei [1]. Due to the very short lifetime of exotic nuclei, this study cannot be performed through usual spectroscopic techniques and one must resort to indirect methods. Breakup is one of these methods. In this reaction, the projectile under analysis dissociates into more elementary components through its interaction with a target. Many such experiments have been performed with the hope to probe exotic nuclear structures far from stability [2], 3].

In order to get valuable information from breakup measurements, one must have not only a fair description of the projectile, but also an accurate reaction model. At present, a fully microscopic description of the reaction is computationally unfeasible. Simplifying assumptions are necessary. First, we will discuss only elastic breakup, i.e. a dissociation process leaving the target unchanged in its ground state. Other channels are simulated through the use of optical potentials. Second, we assume a cluster structure for the projectile. The projectile ground state is assumed to be a bound state of the clusters appearing during the breakup reaction. The bound and continuum states of the projectile are thus described by a few-body Hamiltonian involving effective forces between the constituent clusters. Theoretical reaction models are therefore based on this cluster description of the projectile and effective cluster-cluster and cluster-target interactions.

Even within these simplifying model assumptions, a direct resolution of the resulting many-body Schrödinger equation is still not possible in most cases. In this article, we thus review various approximations that have been developed up to now.

We begin with the models based on the semiclassical approximation [4] in which the projectile-target relative motion is described by a classical trajectory. This approximation is valid at high energies. It leads to the resolution of a time-dependent Schrödinger equation. In a primary version, the time-dependent equation was solved at the first order of the perturbation theory [4]. Then, as computers became more powerful, it could be solved numerically [5, 6, 7, 8, 9, 10]. We present both versions indicating their respective advantages and drawbacks.

We then describe the eikonal approximation [11] and its variants. The principle is to calculate the deviations from a plane-wave motion which are assumed to be weak at high energy. By comparison with the semiclassical model, it is possible to derive the dynamical eikonal approximation (DEA) that combines the advantages of both models [12, 13]. The standard eikonal approximation is obtained by making the additional adiabatic or sudden approximation, which neglects the excitation energies of the projectile. With this stronger simplifying assumption, the final state only differs from the initial bound state by a phase factor. This approach is mostly used to model reactions on light targets at intermediate and high energies. Its drawback is that the Coulomb interaction leads to a divergence of breakup cross sections at forward angles. This problem can be solved using a first-order correction of the Coulomb treatment within the eikonal treatment. A satisfactory approximation of the DEA can then be derived [14, 15]: the Coulomb-corrected eikonal approxima- 
tion (CCE), which remains valid for breakup on heavy targets. It reproduces most of the results of the DEA, although its computational time is significantly lower [16] which is important for the study of the breakup of three-cluster projectiles.

Finally, we present the continuum-discretized coupled-channel method (CDCC) [17, 18], in which the full projectile-target Schrödinger equation is solved approximately, by representing the continuum of the projectile with square-integrable states. This model leads to the numerical resolution of coupled-channel equations, and is suited for low- as high-energy reactions.

All the aforementioned models have been developed initially for two-body projectiles. However, the physics of three-cluster systems, like two-neutron halo nuclei, is the focus of many experimental studies and must also be investigated with these models. We review here the various efforts that have been made in the past few years to extend breakup models to three-cluster projectiles [19, 20, 21].

In Sec. 2, we specify the general theoretical framework within which the projectile is described. The semiclassical model and approximate resolutions of the timedependent Schrödinger equation are described in Sec. 3 Sec.4 4 presents the eikonal approximation as well as the related DEA and CCE models. Next, in Sec. 5, the CDCC method is developed. In Sec. 6 we review applications of breakup reactions to two-body projectiles. In particular, we emphasize the use of breakup to study nuclei far from stability and as an indirect way to infer cross sections of reactions of astrophysical interest. Sec. 7 details the recent efforts made to extend various reaction models to three-body projectiles. We end this review by presenting some views of the future in breakup-reaction theory.

\section{Projectile and reaction models}

We consider the reaction of a projectile $P$ of mass $m_{P}$ and charge $Z_{P} e$ impinging on a target $T$ of mass $m_{T}$ and charge $Z_{T} e$. The projectile is assumed to exhibit a structure made of $N$ clusters with masses $m_{i}$ and charges $Z_{i} e\left(m_{P}=\sum_{i} m_{i}\right.$ and $Z_{P}=\sum_{i} Z_{i}$ ). Its internal properties are described by a Hamiltonian $H_{0}$, depending on a set of $N-1$ internal coordinates collectively represented by notation $\xi$. With the aim of preserving the generality of the presentation of the reaction models, we do not specify here the expression of $H_{0}$. Details are given in Secs. 6 and 7 where applications for the breakup of two- and three-body projectiles are presented.

The states of the projectile are thus described by the eigenstates of $H_{0}$. For total angular momentum $J$ and projection $M$, they are defined by

$$
H_{0} \phi_{\tau}^{J M}(E, \xi)=E \phi_{\tau}^{J M}(E, \xi),
$$

where $E$ is the energy in the projectile centre-of-mass (c.m.) rest frame with respect to the dissociation threshold into $N$ clusters. Index $\tau$ symbolically represents the set of all additional quantum numbers that depend on the projectile structure, like spins and relative orbital momenta of the clusters. Its precise definition depends on the 
number of clusters and on the model selected when defining $H_{0}$. We assume these numbers to be discrete, though some may be continuous in some representations when there are more than two clusters. To simplify the notation, the parity $\pi$ of the eigenstates of $H_{0}$ is understood. In the following, any sum over $J$ implicitly includes a sum over parity.

The negative-energy solutions of Eq. 2.11 correspond to the bound states of the projectile. They are normed to unity. The positive-energy states describe the brokenup projectile with full account of the interactions between the clusters. They are orthogonal and normed according to $\left\langle\phi_{\tau^{\prime}}^{J M}\left(E^{\prime}, \xi\right) \mid \phi_{\tau}^{J M}(E, \xi)\right\rangle=\delta\left(E-E^{\prime}\right) \delta_{\tau \tau^{\prime}}$. To describe final states when evaluating breakup cross sections, we also consider the incoming scattering states $\phi_{\hat{k}_{\xi}}^{(-)}$. They correspond to positive-energy states of $H_{0}$ describing the $N$ clusters moving away from each other in the projectile c.m. frame with specific asymptotic momenta and spin projections. These momenta are not independent, since the sum of the asymptotic kinetic energies of the clusters is the positive energy $E$. However, within that condition, their directions and, if $N>2$, their norms can vary. By $\hat{k}_{\xi}$, we symbolically denote these directions and wave numbers, as well as the projections of the spins of the clusters. These incoming scattering states are thus solutions of the Schrödinger equation

$$
H_{0} \phi_{\hat{k}_{\xi}}^{(-)}(E, \xi)=E \phi_{\hat{k}_{\xi}}^{(-)}(E, \xi) .
$$

They can be expanded into a linear combination of the eigenstates $\phi_{\tau}^{J M}$ of Eq. 2.1) with the same energy as

$$
\phi_{\hat{k}_{\xi}}^{(-)}(E, \xi)=\sum_{J M \tau} a_{\tau}^{J M}\left(\hat{k}_{\xi}\right) \phi_{\tau}^{J M}(E, \xi)
$$

where the coefficients $a_{\tau}^{J M}$ depend on the projectile structure. These scattering states are normed following $\left\langle\phi_{\hat{k}_{\xi}^{\prime}}^{(-)}\left(E^{\prime}, \xi\right) \mid \phi_{\hat{k}_{\xi}}^{(-)}(E, \xi)\right\rangle=\delta\left(E-E^{\prime}\right) \delta\left(\hat{k}_{\xi}-\hat{k}_{\xi}^{\prime}\right)$.

The interactions between the projectile constituents and the target are usually simulated by optical potentials chosen in the literature or obtained by a folding procedure. Within this framework the description of the reaction reduces to the resolution of an $(N+1)$-body Schrödinger equation

$$
\left[\frac{P^{2}}{2 \mu}+H_{0}+V_{P T}(\xi, \mathbf{R})\right] \Psi(\xi, \mathbf{R})=E_{\mathrm{T}} \Psi(\xi, \mathbf{R}),
$$

where $\mathbf{R}=\left(R, \Omega_{R}\right)=\left(R, \theta_{R}, \varphi_{R}\right)$ is the coordinate of the projectile centre of mass relative to the target, $\mathbf{P}$ is the corresponding momentum, $\mu=m_{P} m_{T} /\left(m_{P}+m_{T}\right)$ is the projectile-target reduced mass, and $E_{\mathrm{T}}$ is the total energy in the projectiletarget c.m. frame. The projectile-target interaction $V_{P T}$ is expressed as the sum of the optical potentials (including Coulomb) that simulate the interactions between the projectile constituents and the target, 


$$
V_{P T}(\xi, \mathbf{R})=\sum_{i=1}^{N} V_{i T}\left(R_{i T}\right)
$$

where $\mathbf{R}_{i T}$ is the relative coordinate of the projectile cluster $i$ with respect to the target.

The projectile being initially bound in the state $\phi_{\tau_{0}}^{J_{0} M_{0}}$ of negative energy $E_{0}$, we look for solutions of Eq. (2.4) with an incoming part behaving asymptotically as

$$
\Psi(\xi, \mathbf{R}) \underset{Z \rightarrow-\infty}{\longrightarrow} e^{i\{K Z+\eta \ln [K(R-Z)]\}} \phi_{\tau_{0}}^{J_{0} M_{0}}\left(E_{0}, \xi\right)
$$

where $Z$ is the component of $\mathbf{R}$ in the incident-beam direction. The wavenumber $K$ of the projectile-target relative motion is related to the total energy $E_{\mathrm{T}}$ by

$$
E_{\mathrm{T}}=\frac{\hbar^{2} K^{2}}{2 \mu}+E_{0}
$$

The $P$ - $T$ Sommerfeld parameter is defined as

$$
\eta=Z_{P} Z_{T} e^{2} / \hbar v
$$

where $v=\hbar K / \mu$ is the initial $P-T$ relative velocity.

A first idea that may come to mind is to solve Eq. (2.4) exactly, e.g., within the Faddeev framework or its extensions. However, the infinite range of the Coulomb interaction between the projectile and the target renders the standard equations ill-defined. Only recently significant progress has been made. For example, in Refs. [22, 23], this problem is tackled by using an appropriate screening of the Coulomb force. This technique has been used to successfully describe the elastic scattering and breakup of the deuteron on various targets. However, it has long been limited to light targets (see Ref. [24] for a recent extension to a heavier target). To obtain a model that is valid for all types of target, one must still resort to approximations in the resolution of Eq. (2.4). These approximations are made in the treatment of the projectile-target relative motion, like in the semiclassical (Sec. 3) or eikonal (Sec. 4) approximations, or by using a discretized continuum, like in the CDCC method (Sec. 5).

\section{Semiclassical approximation}

\subsection{Time-dependent Schrödinger equation}

The semiclassical approximation relies on the hypothesis that the projectile-target relative motion can be efficiently described by a classical trajectory $\mathbf{R}(t)$ [4]. It is thus valid when the de Broglie wavelength is small with respect to the impact parameter $b$ characterizing the trajectory, $K b \gg 1$, i.e. when the energy is large enough. 
Along that trajectory, the projectile experiences a time-dependent potential $V$ that simulates the Coulomb and nuclear fields of the target. The internal structure of the projectile, on the contrary, is described quantum-mechanically by the Hamiltonian $H_{0}$. This semiclassical approximation leads to the resolution of the time-dependent equation

$$
i \hbar \frac{\partial}{\partial t} \Psi(\xi, \mathbf{b}, t)=\left[H_{0}+V(\xi, t)\right] \Psi(\xi, \mathbf{b}, t) .
$$

The time-dependent potential is obtained from the difference between the projectiletarget interaction $V_{P T}(2.5)$ and the potential $V_{\text {traj }}$ that defines the classical trajectory

$$
V(\xi, t)=V_{P T}[\xi, \mathbf{R}(t)]-V_{\text {traj }}[R(t)] .
$$

The potential $V_{\text {traj }}$ acts as a $P-T$ scattering potential that bends the trajectory, but does not affect the projectile internal structure. Its interest lies in the fact that $V$ decreases faster than $V_{P T}$. Its effect amounts to changing the phase of the wave function. Usually it is chosen to be the Coulomb potential between the projectile centre of mass and the target, but it may include a nuclear component. At sufficiently high energy, the trajectory is often approximated by a straight line.

For each impact parameter $b$, Eq. 3.1) has to be solved with the initial condition that the projectile is in its ground state,

$$
\Psi^{\left(M_{0}\right)}(\xi, \mathbf{b}, t) \underset{t \rightarrow-\infty}{\longrightarrow} \phi_{\tau_{0}}^{J_{0} M_{0}}\left(E_{0}, \xi\right) .
$$

For each trajectory, the time-dependent wave function $\Psi^{\left(M_{0}\right)}$ must be calculated for the different possible values of $M_{0}$.

\subsection{Cross sections}

From the output of the resolution of Eq. 3.1), the probability of being in a definite state of the projectile can be obtained by projecting the final wave function onto the corresponding eigenstate of $H_{0}$. One can for example compute the elastic scattering probability

$$
P_{\mathrm{el}}(b)=\frac{1}{2 J_{0}+1} \sum_{M_{0}} \sum_{M_{0}^{\prime}}\left|\left\langle\phi_{\tau_{0}}^{J_{0} M_{0}^{\prime}}\left(E_{0}, \xi\right) \mid \Psi^{\left(M_{0}\right)}(\xi, \mathbf{b}, t \rightarrow+\infty)\right\rangle\right|^{2} .
$$

This probability depends only on the norm of the impact parameter $b$ because the time-dependent wave function $\Psi^{\left(M_{0}\right)}$ depends on the orientation of $\mathbf{b}$, i.e. on the azimuthal angle $\varphi_{R}$, only through a phase that cancels out in the calculation of $P_{\mathrm{el}}$. From this probability, the cross section for the elastic scattering in direction $\Omega$ is obtained as 


$$
\frac{d \sigma_{\mathrm{el}}}{d \Omega}=\frac{d \sigma_{\mathrm{el}}^{\mathrm{traj}}}{d \Omega} P_{\mathrm{el}}[b(\Omega)]
$$

where $b(\Omega)$ is given by the classical relation between the scattering angle and the impact parameter derived from potential $V_{\text {traj. }}$. The factor $d \sigma_{\mathrm{el}}^{\text {traj }} / d \Omega$ is the elastic scattering cross section obtained from $V_{\text {traj. }}$. In most cases $d \sigma_{\mathrm{el}}^{\text {traj }} / d \Omega$ is generated from the Coulomb interaction and is thus the $P-T$ Rutherford cross section.

Likewise, a general breakup probability density can be computed by projecting the final wave function onto the ingoing scattering states of $H_{0}$,

$$
\frac{d P_{\mathrm{bu}}}{d \hat{k}_{\xi} d E}(b)=\frac{1}{2 J_{0}+1} \sum_{M_{0}}\left|\left\langle\phi_{\hat{k}_{\xi}}^{(-)}(E, \xi) \mid \Psi^{\left(M_{0}\right)}(\xi, \mathbf{b}, t \rightarrow+\infty)\right\rangle\right|^{2} .
$$

After integration and summation over $\hat{k}_{\xi}$, the breakup probability per unit energy reads

$$
\frac{d P_{\mathrm{bu}}}{d E}(b)=\frac{1}{2 J_{0}+1} \sum_{M_{0}} \sum_{J M \tau}\left|\left\langle\phi_{\tau}^{J M}(E, \xi) \mid \Psi^{\left(M_{0}\right)}(\xi, \mathbf{b}, t \rightarrow+\infty)\right\rangle\right|^{2} .
$$

Similarly to Eq. 3.5), a differential cross section for the breakup of the projectile is given by

$$
\frac{d \sigma_{\mathrm{bu}}}{d E d \Omega}=\frac{d \sigma_{\mathrm{el}}^{\mathrm{traj}}}{d \Omega} \frac{d P_{\mathrm{bu}}}{d E}[b(\Omega)] .
$$

The breakup cross section can then be obtained by summing the breakup probability over all impact parameters

$$
\frac{d \sigma_{\mathrm{bu}}}{d E}=2 \pi \int_{0}^{\infty} \frac{d P_{\mathrm{bu}}}{d E}(b) b d b
$$

Because of the trajectory hypothesis of the semiclassical approximation, the impact parameter $b$ is a classical variable. Therefore, no interference between the different trajectories can appear. This is the major disadvantage of that technique since quantal interferences can play a significant role in reactions, in particular in those which are nuclear dominated.

\subsection{Resolution at the first order of the perturbation theory}

In the early years of the semiclassical approximations, Eq. 3.1) was solved at the first order of the perturbation theory [4]. This technique, due to Alder and Winther, was applied to analyze the first Coulomb-breakup experiments of halo nuclei [25].

The time-dependent wave function $\Psi^{\left(M_{0}\right)}$ is expanded upon the basis of eigenstates of $H_{0}$ in Eq. (2.1). At the first order of the perturbation theory, the resulting 
equation is solved by considering that $V$ is small. With the initial condition (3.3), the wave function at first order is given by [4, 26]

$$
e^{\frac{i}{\hbar} H_{0} t} \Psi^{\left(M_{0}\right)}(\xi, \mathbf{b}, t)=\left[1+\frac{1}{i \hbar} \int_{-\infty}^{t} e^{\frac{i}{\hbar} H_{0} t^{\prime}} V\left(\xi, t^{\prime}\right) e^{-\frac{i}{\hbar} H_{0} t^{\prime}} d t^{\prime}\right] \phi_{\tau_{0}}^{J_{0} M_{0}}\left(E_{0}, \xi\right) .
$$

Following Eq. (3.6), the general breakup probability density reads

$$
\frac{d P_{\mathrm{bu}}}{d \hat{k}_{\xi} d E}(b)=\frac{\hbar^{-2}}{2 J_{0}+1} \sum_{M_{0}}\left|\int_{-\infty}^{+\infty} e^{i \omega t}\left\langle\phi_{\hat{k}_{\xi}}^{(-)}(E, \xi)|V(\xi, t)| \phi_{\tau_{0}}^{J_{0} M_{0}}\left(E_{0}, \xi\right)\right\rangle d t\right|^{2},
$$

where $\omega=\left(E-E_{0}\right) / \hbar$. The breakup probability per unit energy reads

$$
\frac{d P_{\mathrm{bu}}}{d E}(b)=\frac{\hbar^{-2}}{2 J_{0}+1} \sum_{M_{0}} \sum_{J M \tau}\left|\int_{-\infty}^{+\infty} e^{i \omega t}\left\langle\phi_{\tau}^{J M}(E, \xi)|V(\xi, t)| \phi_{\tau_{0}}^{J_{0} M_{0}}\left(E_{0}, \xi\right)\right\rangle d t\right|^{2}
$$

With Eq. 3.10), exact expressions can be calculated when considering a purely Coulomb $P$ - $T$ interaction for straight-line trajectories in the far-field approximation [27], i.e. by assuming that the charge densities of the projectile and target do not overlap during the collision. One obtains

$$
\begin{aligned}
& \left\langle\phi_{\tau}^{J M}(E, \xi) \mid \Psi^{\left(M_{0}\right)}(\xi, \mathbf{b}, t \rightarrow+\infty)\right\rangle= \\
& Z_{T} e \frac{e^{-i E t / \hbar}}{i \hbar} \sum_{\lambda \mu} \frac{4 \pi}{2 \lambda+1} I_{\lambda \mu}(\omega, b)\left\langle\phi_{\tau}^{J M}(E, \xi)\left|\mathscr{M}_{\mu}^{\mathrm{E} \lambda}(\xi)\right| \phi_{\tau_{0}}^{J_{0} M_{0}}\left(E_{0}, \xi\right)\right\rangle,
\end{aligned}
$$

where $\mathscr{M}_{\mu}^{\mathrm{E} \lambda}$ are the electric multipoles operators of rank $\lambda$, and $I_{\lambda \mu}$ are time integrals (see, e.g., Eq. (13) of Ref. [28]) that can be evaluated analytically as [27]

$$
I_{\lambda \mu}(\omega, b)=\sqrt{\frac{2 \lambda+1}{\pi}} \frac{1}{v} \frac{i^{\lambda+\mu}}{\sqrt{(\lambda+\mu) !(\lambda-\mu) !}}\left(-\frac{\omega}{v}\right)^{\lambda} K_{|\mu|}\left(\frac{\omega b}{v}\right),
$$

where $K_{n}$ is a modified Bessel function [29].

If only the dominant dipole term E1 of the interaction is considered, the breakup probability (3.12) reads [30]

$$
\begin{aligned}
& \frac{d P_{\mathrm{bu}}^{\mathrm{E} 1}}{d E}(b)=\frac{16 \pi}{9}\left(\frac{Z_{T} e}{\hbar v}\right)^{2} \\
& \times\left(\frac{\omega}{v}\right)^{2}\left[K_{1}^{2}\left(\frac{\omega b}{v}\right)+K_{0}^{2}\left(\frac{\omega b}{v}\right)\right] \frac{d B(\mathrm{E} 1)}{d E} .
\end{aligned}
$$

The last factor is the dipole strength function per energy unit [30],

$$
\frac{d B(\mathrm{E} 1)}{d E}=\frac{1}{2 J_{0}+1} \sum_{\mu M_{0}} \sum d \hat{k}_{\xi}\left|\left\langle\phi_{\hat{k}_{\xi}}^{(-)}(E, \xi)\left|\mathscr{M}_{\mu}^{\mathrm{E} 1}(\xi)\right| \phi_{\tau_{0}}^{J_{0} M_{0}}\left(E_{0}, \xi\right)\right\rangle\right|^{2}
$$




$$
=\frac{1}{2 J_{0}+1} \sum_{\mu M_{0}} \sum_{J M \tau}\left|\left\langle\phi_{\tau}^{J M}(E, \xi)\left|\mathscr{M}_{\mu}^{\mathrm{E} 1}(\xi)\right| \phi_{\tau_{0}}^{J_{0} M_{0}}\left(E_{0}, \xi\right)\right\rangle\right|^{2} .
$$

Since modified Bessel functions decrease exponentially, the asymptotic behaviour of $d P_{\mathrm{bu}}^{\mathrm{E} 1} / d E$ for $b \rightarrow \infty$ is proportional to $\exp (-2 \omega b / v)$.

In the case of a purely Coulomb $P-T$ interaction, the first order of the perturbation theory exhibits many appealing aspects. First, it can be solved analytically. Second, the dynamics part $\left(I_{\lambda \mu}\right)$ and structure part (matrix elements of $\mathscr{M}_{\mu}^{\mathrm{E} \lambda}$ ) are separated in the expression of the breakup amplitudes 3.13). This first-order approximation has therefore often been used to analyze Coulomb-breakup experiments by assuming pure E1 breakup (see, e.g., Ref. [25]). However, as will be seen later, higher-order and nuclear-interaction effects are usually not negligible, and a proper analysis of experimental data requires a more sophisticated approximation.

\subsection{Numerical resolution}

The time-dependent Schrödinger equation can also be solved numerically. Various groups have developed algorithms for that purpose [5, 6, 7, 8, 9, 10, 31, 32]. They make use of an approximation of the evolution operator $U$ applied iteratively to the initial bound state wave function following the scheme

$$
\Psi^{\left(M_{0}\right)}(\xi, \mathbf{b}, t+\Delta t)=U(t+\Delta t, t) \Psi^{\left(M_{0}\right)}(\xi, \mathbf{b}, t) .
$$

Although higher-order algorithms exist (see, e.g., Ref. [33]), all practical calculations are performed with second-order approximations of $U$. Various expressions of this approximation exist, depending mainly on the way of representing the timedependent projectile wave function. However they are in general similar to [10]

$$
U(t+\Delta t)=e^{-i \frac{\Delta t}{2 \hbar} V(\xi, t+\Delta t)} e^{-i \frac{\Delta t}{\hbar} H_{0}} e^{-i \frac{\Delta t}{2 \hbar} V(\xi, t)}+\mathscr{O}\left(\Delta t^{3}\right) .
$$

With this expression, the time-dependent potential can be treated separately from the time-independent Hamiltonian $H_{0}$, which greatly simplifies the calculation of the time evolution when the wave functions are discretized on a mesh [10].

The significant advantage of this technique over the first order of perturbation is that it naturally includes higher-order effects. Moreover, the nuclear interaction between the projectile and the target can be easily added in the numerical scheme [34]. However, the dynamical and structure evolutions being now more deeply entangled, the analysis of the numerical resolution of the Schrödinger equation is less straightforward than its first-order approximation. The numerical technique is also much more time-consuming than the perturbation one. The first order of the perturbation theory therefore remains a useful tool to qualitatively analyze calculations of Coulomb-dominated reactions performed with more elaborate models. Moreover, as will be seen in Sec. 4.4, it can be used to correct the erroneous treatment of the Coulomb interaction within the eikonal description of breakup reactions. 
Fig. 1 illustrates the numerical resolution of the time-dependent Schrödinger equation for the Coulomb breakup of ${ }^{11} \mathrm{Be}$ on lead at $68 \mathrm{MeV}$ /nucleon [10]. It shows the breakup cross section as a function of the relative energy $E$ between the ${ }^{10} \mathrm{Be}$ core and the halo neutron after dissociation. The full line corresponds to the calculation with both Coulomb and nuclear $P-T$ interactions. The dashed line is the result for a purely Coulomb potential, in which the nuclear interaction is simulated by an impact parameter cutoff at $b_{\min }=13 \mathrm{fm}$. A calculation performed with an impact parameter cutoff at $b_{\min }=30 \mathrm{fm}$ simulating a forward-angle cut is plotted as a dotted line. The experimental data from Ref. [25] are multiplied by a factor of 0.85 as suggested in Ref. [35] after a remeasurement.

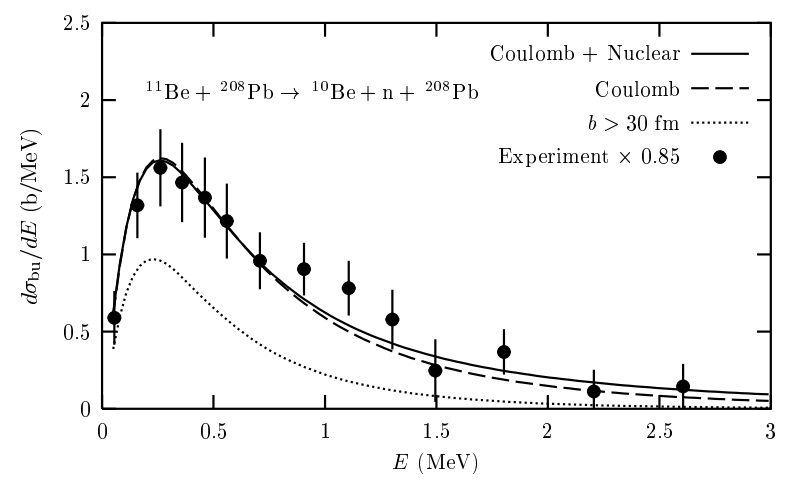

Fig. 1 Breakup cross section of ${ }^{11} \mathrm{Be}$ on $\mathrm{Pb}$ at $68 \mathrm{MeV} /$ nucleon as a function of the relative energy $E$ between the ${ }^{10} \mathrm{Be}$ core and the neutron. Calculations are performed within the semiclassical approximation with or without nuclear interaction [10]. Experimental data [25] are scaled by 0.85 [35]. Reprinted figure with permission from Ref. [10]. Copyright (2003) by the American Physical Society.

This example shows the validity of the semiclassical approximation to describe breakup observables in the projectile c.m. frame for collisions at intermediate energies. It also confirms that for heavy targets the reaction is strongly dominated by the Coulomb interaction. The inclusion of optical potentials to simulate the nuclear $P-T$ interactions indeed only slightly increases the breakup cross section at large energy. This shows that Coulomb-breakup calculations are not very sensitive to the uncertainty related to the choice of the optical potentials. Nevertheless, since optical potentials can be very easily included in the numerical resolution of the timedependent Schrödinger equation, they should be used so as to avoid the imprecise impact-parameter cutoff necessary in purely Coulomb calculations. 


\section{Eikonal approximations}

\subsection{Dynamical eikonal approximation}

Let us now turn to a purely quantal treatment providing approximate solutions of the Schrödinger equation (2.4). At sufficiently high energy, the projectile is only slightly deflected by the target. The dominant dependence of the $(N+1)$-body wave function $\Psi$ on the projectile-target coordinate $\mathbf{R}$ is therefore in the plane wave contributing to the incident relative motion (2.6). The main idea of the eikonal approximation is to factorize that plane wave out of the wave function to define a new function $\widehat{\Psi}$ whose variation with $\mathbf{R}$ is expected to be small [11, 30, 36]

$$
\Psi(\xi, \mathbf{R})=e^{i K Z} \widehat{\Psi}(\xi, \mathbf{R}) .
$$

With factorization (4.1) and energy conservation (2.7), the Schrödinger equation (2.4) becomes

$$
\left[\frac{P^{2}}{2 \mu}+v P_{Z}+H_{0}-E_{0}+V_{P T}(\xi, \mathbf{R})\right] \widehat{\Psi}(\xi, \mathbf{R})=0,
$$

where the relative velocity $v$ between projectile and target is assumed to be large.

The first step in the eikonal approximation is to assume the second-order derivative $P^{2} / 2 \mu$ negligible with respect to the first-order derivative $\nu P_{Z}$,

$$
\frac{P^{2}}{2 \mu} \widehat{\Psi}(\xi, \mathbf{R}) \ll v P_{Z} \widehat{\Psi}(\xi, \mathbf{R}) .
$$

This first step leads to the second-order equation (but now first-order in $Z$ ),

$$
i \hbar v \frac{\partial}{\partial Z} \widehat{\Psi}(\xi, \mathbf{b}, Z)=\left[H_{0}-E_{0}+V_{P T}(\xi, \mathbf{R})\right] \widehat{\Psi}(\xi, \mathbf{b}, Z),
$$

where the dependence of the wave function on the longitudinal $Z$ and transverse b parts of the projectile-target coordinate $\mathbf{R}$ has been made explicit. This equation is mathematically equivalent to the time-dependent Schrödinger equation (3.1) for straight-line trajectories with $t$ replaced by $Z / v$. It can thus be solved using any of the algorithms cited in Sec. 3.4. However, contrary to time-dependent models, it is obtained without the semiclassical approximation. The projectile-target coordinate components $\mathbf{b}$ and $Z$ are thus quantal variables. Interferences between solutions obtained at different $b$ values are thus taken here into account. This first step is known as the dynamical eikonal approximation (DEA) [12, 13]. 


\subsection{Cross sections}

The transition matrix element for elastic scattering into direction $\Omega=(\theta, \varphi)$ of the final momentum $\mathbf{K}=(K, \Omega)$ of the projectile in the c.m. frame reads [37]

$$
T_{f i}=\left\langle e^{i \mathbf{K} \cdot \mathbf{R}} \phi_{\tau_{0}}^{J_{0} M_{0}^{\prime}}\left(E_{0}, \xi\right)\left|V_{P T}(\xi, \mathbf{R})\right| \Psi^{\left(M_{0}\right)}(\xi, \mathbf{R})\right\rangle
$$

where $\Psi^{\left(M_{0}\right)}$ is the exact solution of the Schrödinger equation 2.4 with the asymptotic condition (2.6). By using Eqs. (4.1), 2.1), and (4.4), one obtains the approximation [12]

$$
\begin{aligned}
T_{f i} & =\left\langle e^{i \mathbf{K} \cdot \mathbf{R}} \phi_{\tau_{0}}^{J_{0} M_{0}^{\prime}}\left(E_{0}, \xi\right)\left|e^{i K Z}\left[H_{0}-E_{0}+V_{P T}(\xi, \mathbf{R})\right]\right| \widehat{\Psi}^{\left(M_{0}\right)}(\xi, \mathbf{R})\right\rangle \\
& \approx i \hbar v \int d \mathbf{R} e^{-i \mathbf{q} \cdot \mathbf{b}} \frac{\partial}{\partial Z}\left\langle\phi_{\tau_{0}}^{J_{0} M_{0}^{\prime}}\left(E_{0}, \xi\right) \mid \widehat{\Psi}^{\left(M_{0}\right)}(\xi, \mathbf{R})\right\rangle
\end{aligned}
$$

where the transfered momentum $\mathbf{q}=\mathbf{K}-K \hat{\mathbf{Z}}$ is assumed to be purely transverse, i.e. $\exp [i(\mathbf{K} \cdot \hat{\mathbf{Z}}-K)]$, is neglected. The norm of $q$ is linked to the scattering angle by

$$
q=2 K \sin \theta / 2
$$

Let us define the elastic amplitude

$$
S_{\mathrm{el}, M_{0}^{\prime}}^{\left(M_{0}\right)}(\mathbf{b})=\left\langle\phi_{\tau_{0}}^{J_{0} M_{0}^{\prime}}\left(E_{0}, \xi\right) \mid \widehat{\Psi}^{\left(M_{0}\right)}(\xi, \mathbf{b}, Z \rightarrow+\infty)\right\rangle-\delta_{M_{0}^{\prime} M_{0}} .
$$

The transition matrix element (4.6) reads after integration over $Z$,

$$
T_{f i}=i \hbar v \int d \mathbf{b} e^{-i \mathbf{q} \cdot \mathbf{b}} e^{i\left(M_{0}-M_{0}^{\prime}\right) \varphi_{R}} S_{\mathrm{el}, M_{0}^{\prime}}^{\left(M_{0}\right)}(b \hat{\mathbf{X}})
$$

where $\varphi_{R}$ is the azimuthal angle characterizing $\mathbf{b}$. The phase factor $\exp \left[i\left(M_{0}-\right.\right.$ $\left.\left.M_{0}^{\prime}\right) \varphi_{R}\right]$ arises from the rotation of the wave functions when the orientation of $\mathbf{b}$ varies [13]. The integral over $\varphi_{R}$ can be performed analytically, which leads to the following expression for the elastic differential cross section [13]

$$
\frac{d \sigma_{\mathrm{el}}}{d \Omega}=K^{2} \frac{1}{2 J_{0}+1} \sum_{M_{0} M_{0}^{\prime}}\left|\int_{0}^{\infty} b d b J_{\left|M_{0}-M_{0}^{\prime}\right|}(q b) S_{\mathrm{el}, M_{0}^{\prime}}^{\left(M_{0}\right)}(b \hat{\mathbf{X}})\right|^{2},
$$

where $J_{m}$ is a Bessel function [29]. From Eq. 44.10], one can see that contrary to the semiclassical approximation (3.5), the eikonal elastic cross section is obtained as a coherent sum of elastic amplitudes over all $b$ values. This illustrates that quantum interferences are taken into account in the eikonal framework.

The transition matrix element for dissociation reads

$$
T_{f i}=\left\langle e^{i \mathbf{K}^{\prime} \cdot \mathbf{R}} \phi_{\hat{k}_{\xi}}^{(-)}(E, \xi)\left|V_{P T}(\xi, \mathbf{R})\right| \Psi^{\left(M_{0}\right)}(\xi, \mathbf{R})\right\rangle,
$$


where $\mathbf{K}^{\prime}=\left(K^{\prime}, \Omega\right)$ is the final projectile-target wave vector. One can then proceed as for the elastic scattering. Using Eqs. (4.1), 2.2), and 4.4, taking into account the energy conservation,

$$
\frac{\hbar^{2} K^{2}}{2 \mu}+E_{0}=\frac{\hbar^{2} K^{\prime 2}}{2 \mu}+E
$$

and assuming the transfered momentum $\mathbf{q}=\mathbf{K}^{\prime}-K \hat{\mathbf{Z}}$ to be purely transverse, the transition matrix element is expressed as

$$
T_{f i} \approx i \hbar v \int d \mathbf{b} e^{-i \mathbf{q} \cdot \mathbf{b}} S_{\mathrm{bu}}^{\left(M_{0}\right)}\left(E, \hat{k}_{\xi}, \mathbf{b}\right),
$$

with the breakup amplitude

$$
S_{\mathrm{bu}}^{\left(M_{0}\right)}\left(E, \hat{k}_{\xi}, \mathbf{b}\right)=\left\langle\phi_{\hat{k}_{\xi}}^{(-)}(E, \xi) \mid \widehat{\Psi}^{\left(M_{0}\right)}(\xi, \mathbf{b}, Z \rightarrow+\infty)\right\rangle .
$$

The differential cross section for breakup is given by

$$
\frac{d \sigma}{d \hat{k}_{\xi} d E d \Omega} \propto \frac{1}{2 J_{0}+1} \sum_{M_{0}}\left|\int d \mathbf{b} e^{-i \mathbf{q} \cdot \mathbf{b}} S_{\mathrm{bu}}^{\left(M_{0}\right)}\left(E, \hat{k}_{\xi}, \mathbf{b}\right)\right|^{2},
$$

where the proportionality factor depends on the phase space. Like the elastic scattering cross section (4.10), it is obtained from a coherent sum of breakup amplitudes (4.14), confirming the quantum-mechanical character of the eikonal approximation. Here also, the integral over $\varphi_{R}$ can be performed analytically and leads to Bessel functions [13].

By integrating expression (4.15) over unmeasured quantities, one can obtain the breakup cross sections with respect to the desired variables, like the internal excitation energy of the projectile. Since these operations depend on the projectile internal structure, we delay the presentation of some detailed expressions to Secs. 6 and 7 treating of two-body [13] and three-body [19] breakup.

\subsection{Standard eikonal approximation}

In most references, the concept of eikonal approximation involves a further simplification to the DEA [38, 30]. This adiabatic, or sudden, approximation consists in neglecting the excitation energy of the projectile compared to the incident kinetic energy. It comes down to assume the low-lying spectrum of the projectile to be degenerate with its ground state, i.e. to consider the internal coordinates of the projectile as frozen during the reaction [30]. This approximation therefore holds only for high-energy collisions that occur during a very brief time. This second assumption leads to neglect the term $H_{0}-E_{0}$ in the DEA equation (4.4) which then reads 


$$
i \hbar v \frac{\partial}{\partial Z} \widehat{\Psi}(\xi, \mathbf{b}, Z)=V_{P T}(\xi, \mathbf{R}) \widehat{\Psi}(\xi, \mathbf{b}, Z) .
$$

The solution of Eq. (4.16) that follows the asymptotic condition (2.6) exhibits the well-known eikonal form [11, 36]

$$
\widehat{\Psi}^{\left(M_{0}\right)}(\xi, \mathbf{b}, Z)=\exp \left[-\frac{i}{\hbar v} \int_{-\infty}^{Z} V_{P T}\left(\xi, \mathbf{b}, Z^{\prime}\right) d Z^{\prime}\right] \phi_{\tau_{0}}^{J_{0} M_{0}}\left(E_{0}, \xi\right) .
$$

After the collision, the whole information about the change in the projectile wave function is thus contained in the phase shift

$$
\chi\left(\mathbf{s}_{\xi}, \mathbf{b}\right)=-\frac{1}{\hbar v} \int_{-\infty}^{+\infty} V_{P T}(\xi, \mathbf{R}) d Z .
$$

Due to translation invariance, this eikonal phase $\chi$ depends only on the transverse components $\mathbf{b}$ of the projectile-target coordinate $\mathbf{R}$ and $\mathbf{s}_{\xi}$ of the projectile internal coordinates $\xi$. Cross sections within this standard eikonal approximation are obtained as explained in Sec. 4.2, replacing $\widehat{\Psi}^{\left(M_{0}\right)}$ by $e^{i \chi} \phi_{\tau_{0}}^{J_{0} M_{0}}$.

Being obtained from the adiabatic approximation, expressions (4.17) and (4.18) are valid only for short-range potentials. For the Coulomb interaction, the assumption that the reaction takes place in a short time no longer holds, due to its infinite range. The adiabatic approximation thus fails for Coulomb-dominated reactions [30]. Besides imprecise uses of a cutoff at large impact parameters [39], there are two ways to avoid this problem. The first is not to make the adiabatic approximation, i.e. to resort to the more complicated DEA (see Sec. 4.1). The second is to correct the eikonal phase for the Coulomb interaction as suggested in Ref. [14] (see Sec. 4.4]. Nevertheless, as shown in Ref. [13], the Coulomb divergence does not affect eikonal calculations performed on light targets at high enough energies. Most of the nuclear-dominated reactions can thus be analyzed within an eikonal model including the adiabatic approximation (see, e.g., Ref. [40]).

Fig. 2 2 illustrates the difference between the DEA (full line), the usual eikonal approximation (dashed line) and the semiclassical approximation (dotted line) when Coulomb dominates. It shows the breakup cross section of ${ }^{11} \mathrm{Be}$ on $\mathrm{Pb}$ at 69 $\mathrm{MeV} /$ nucleon for a ${ }^{10} \mathrm{Be}-\mathrm{n}$ relative energy of $0.3 \mathrm{MeV}$ as a function of the $P-T$ scattering angle. As explained above, the usual eikonal approximation diverges for the Coulomb-dominated breakup, i.e. at forward angles. The DEA, which does not include the adiabatic approximation, exhibits a regular behaviour at these angles. Interestingly, the semiclassical approximation follows the general behaviour of the DEA, except for the oscillations due to quantum interferences between different $b$ values. The DEA has therefore the advantage of being valid for describing any breakup observable on both light and heavy targets.

The nuclei studied through breakup reactions being exotic, it may be difficult, if not impossible, to find optical potentials that describe the scattering of the clusters by the target. One way to circumvent that problem is to resort to what is usually known as the Glauber model [42, 38, 30, 36]. This model has been mostly used 


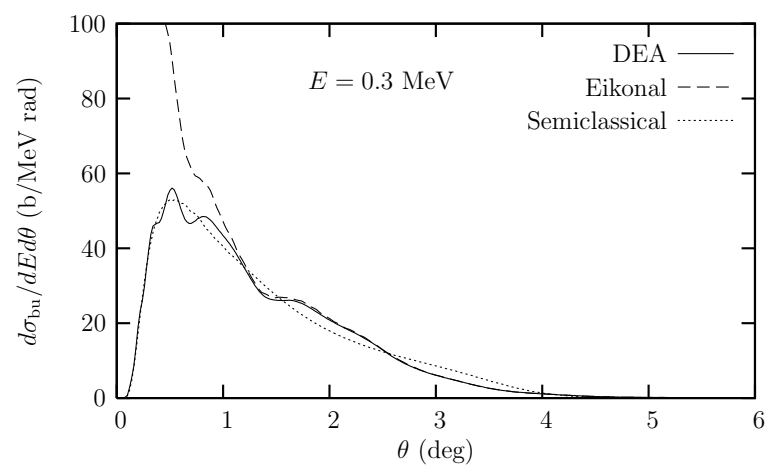

Fig. 2 Breakup cross section of ${ }^{11} \mathrm{Be}$ on $\mathrm{Pb}$ at $69 \mathrm{MeV} /$ nucleon as a function of the $P-T$ scattering angle in the $P-T$ c.m. frame for a ${ }^{10} \mathrm{Be}-\mathrm{n}$ energy $E=0.3 \mathrm{MeV}$. Calculations are performed within the DEA, usual eikonal, and semiclassical approximations [41].

to calculate total and reaction cross sections. At the optical-limit approximation (OLA) of the Glauber model, correlations in the cluster and target wave functions are neglected. The nuclear component of the eikonal phase shift for cluster $i$ is then expressed as a function of the densities $\rho_{T}$ of the target and $\rho_{i}$ of the cluster, and of a profile function $1-e^{i \chi_{\mathrm{NN}}}$ that corresponds to an effective nucleon-nucleon interaction. The nuclear component of the eikonal phase shift is approximated by [30]

$$
\chi_{i}^{N}\left(\mathbf{b}_{i}\right)=i \iint \rho_{T}\left(\mathbf{r}_{T}\right) \rho_{i}\left(\mathbf{r}_{i}\right)\left[1-e^{i \chi_{\mathrm{NN}}\left(\left|\mathbf{b}_{i}-\mathbf{s}_{T}+\mathbf{s}_{i}\right|\right)}\right] d \mathbf{r}_{T} d \mathbf{r}_{i}
$$

where $\mathbf{s}_{T}$ and $\mathbf{s}_{i}$ are the transverse components of the internal coordinates $\mathbf{r}_{T}$ of the target and $\mathbf{r}_{i}$ of cluster $i$, respectively, and $\mathbf{b}_{i}$ is the transverse component of the c.m. coordinate of cluster $i$. The OLA is therefore equivalent to the double-folding of an effective nucleon-nucleon interaction. The density of the target can usually be obtained from experimental data. The cluster density being unknown, it has to be estimated from some structure model, like a mean-field calculation. The profile function is usually parametrized as [30, 43]

$$
1-e^{i \chi_{\mathrm{NN}}(b)}=\frac{1-i \alpha_{\mathrm{NN}}}{4 \pi \beta_{\mathrm{NN}}} \sigma_{\mathrm{NN}}^{\text {tot }} \exp \left(-\frac{b^{2}}{2 \beta_{\mathrm{NN}}}\right),
$$

where $\sigma_{\mathrm{NN}}^{\text {tot }}$ is the total cross section for the N-N collision, $\alpha_{\mathrm{NN}}$ is the ratio of the real part to the imaginary part of the N-N scattering amplitude, and $\beta_{\mathrm{NN}}$ is the slope parameter of the N-N elastic differential cross section. These parameters depend on the nucleon type ( $\mathrm{p}$ or $\mathrm{n}$ ) and on the incident energy. Their values can be found in the literature (see, e.g., Ref. [43]). The validity of the Glauber approximation is discussed in Ref. [44]. 


\subsection{Coulomb-corrected eikonal approximation}

The eikonal approximation gives excellent results for nuclear-dominated reactions [30, 13]. However, as mentioned above, it suffers from a divergence problem when the Coulomb interaction becomes significant. To explain this, let us divide the eikonal phase (4.18) into its Coulomb and nuclear contributions

$$
\chi\left(\mathbf{s}_{\xi}, \mathbf{b}\right)=\chi_{P T}^{C}(b)+\chi^{C}\left(\mathbf{s}_{\xi}, \mathbf{b}\right)+\chi^{N}(\mathbf{s} \xi, \mathbf{b}) .
$$

In this expression, $\chi_{P T}^{C}$ is the global elastic Coulomb eikonal phase between the projectile and the target. However, Coulomb forces not only act globally on the projectile, they also induce 'tidal' effects due to their different actions on the various clusters. The tidal Coulomb phase $\chi^{C}$ is due to the difference between the cluster-target and projectile-target bare Coulomb interactions. The remaining phase $\chi^{N}$ contains effects of the nuclear forces as well as of differences between Coulomb forces taking the finite size of the clusters into account and the bare Coulomb forces.

At the eikonal approximation, the integral (4.18) defining $\chi_{P T}^{C}$ diverges and must be calculated with a cutoff [11, 30]. Up to an additional cutoff-dependent term that plays no role in the cross sections, it can be written as [36]

$$
\chi_{P T}^{C}(b)=2 \eta \ln (K b)
$$

where appears the projectile-target Sommerfeld parameter $\eta$ defined in Eq. (2.8). The phase 4.22) depends only on $b$.

The tidal Coulomb phase is computed with Eq. 4.18) for the difference between the bare Coulomb interactions for the clusters in the projectile and the global $P-T$ Coulomb interaction,

$$
\chi^{C}(\mathbf{s} \xi, \mathbf{b})=-\frac{\eta}{Z_{P}} \int_{-\infty}^{+\infty}\left(\sum_{i=1}^{N} \frac{Z_{i}}{\left|\mathbf{R}_{i T}\right|}-\frac{Z_{P}}{|\mathbf{R}|}\right) d Z .
$$

It can be expressed analytically. Because of the long range of the E1 component of the Coulomb force, this phase behaves as $1 / b$ at large distances [13, 16]. In the calculation of the breakup cross sections 4.15, the integration over $b d b$ diverges for small $q$ values, i.e. at forward angles, because of the corresponding $1 / b$ asymptotic behaviour of the breakup amplitude, as illustrated in Fig. 2] This divergence occurs only in the first-order term $i \chi^{C}$ of the expansion of the eikonal Coulomb amplitude $\exp \left(i \chi^{C}\right)$.

As seen in Sec. 3.3 the first order approximation 3.15) decreases exponentially at large $b$ and hence does not display such a divergence. A plausible correction is therefore to replace the exponential of the eikonal phase according to [14, 15

$$
e^{i \chi} \rightarrow e^{i \chi_{P T}^{C}}\left(e^{i \chi^{C}}-i \chi^{C}+i \chi^{F O}\right) e^{i \chi^{N}}
$$

where $\chi^{F O}$ is the result of first-order perturbation theory 3.10 , 


$$
\chi^{F O}(\xi, \mathbf{b})=-\frac{\eta}{Z_{P}} \int_{-\infty}^{+\infty} e^{i \omega Z / v}\left(\sum_{i=1}^{N} \frac{Z_{i}}{\left|\mathbf{R}_{i T}\right|}-\frac{Z_{P}}{|\mathbf{R}|}\right) d Z .
$$

Note that because of the phase $e^{i \omega Z / v}$, the integrand in Eq. (4.25) does not exhibit a translational invariance. The first-order phase $\chi^{F O}$ depends on all internal coordinates of the projectile. When the adiabatic approximation is applied to Eq. 44.25), i.e. when $\omega$ is set to 0 , one recovers exactly the Coulomb eikonal phase (4.23). This suggests that without adiabatic approximation the first-order term of $\exp \left(i \chi^{C}\right)$ would be $i \chi^{F O}$ (4.25) instead of $i \chi^{C}$ (4.23), intuitively validating the correction (4.24). Furthermore, since a simple analytic expression is available for each of the Coulomb multipoles (see Sec. 3.3), this correction is easy to implement.

With this Coulomb correction, the breakup of loosely-bound projectiles can be described within the eikonal approximation taking on (nearly) the same footing both Coulomb and nuclear interactions at all orders. This approximation has been tested and validated for a two-body projectile in Ref. [16]. Note that in all practical cases [15, 16, 19], only the dipole term of the first-order expansion (3.13) is retained to evaluate $\chi^{F O}$

Fig. 3 illustrates the accuracy of the CCE for the breakup of ${ }^{11} \mathrm{Be}$ on lead at $69 \mathrm{MeV} /$ nucleon [16]. The figure presents the parallel-momentum distribution between the ${ }^{10} \mathrm{Be}$ core and the halo neutron after dissociation. This observable has been computed within the DEA (full line), which serves as a reference calculation, the CCE (dotted line), the eikonal approximation including the adiabatic approximation (dashed line), and the first-order of the perturbation theory (dash-dotted line). The usual eikonal approximation requires a cutoff at large impact parameter to avoid divergence. The value $b_{\max }=71 \mathrm{fm}$ is chosen from the value prescribed in Ref. [39]. At the first order or the perturbation theory, the nuclear interaction is simulated by an impact parameter cutoff at $b_{\min }=15 \mathrm{fm}$.

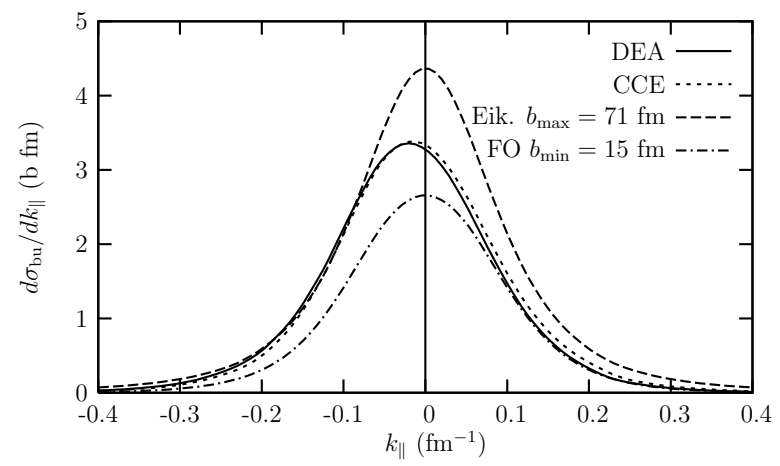

Fig. 3 Breakup of ${ }^{11} \mathrm{Be}$ on $\mathrm{Pb}$ at $69 \mathrm{MeV} /$ nucleon. The parallel-momentum distribution between the ${ }^{10} \mathrm{Be}$ core and the halo neutron is computed within the DEA, the CCE, the eikonal approximation including the adiabatic approximation, and the first-order of the perturbation theory [16]. Reprinted figure with permission from Ref. [16]. Copyright (2008) by the American Physical Society. 
We first see that the magnitude of the CCE cross section is close to the DEA one, whereas, the other two approximations give too large (eikonal) or too small (first order) cross sections. Moreover the CCE reproduces nearly perfectly the shape of the DEA distribution. In particular the asymmetry, due to dynamical effects, is well reproduced. This result suggests that in addition to solving the Coulomb divergence problem introduced by the adiabatic approximation, the CCE also restores some dynamical and higher-order effects missing in its ingredients, the usual eikonal approximation and the first order of the perturbation theory.

\section{Continuum-discretized coupled-channel method}

The CDCC method is a fully quantal approximation which does not imply some restriction on energies. Its main interest lies in low energies where the previous methods are not valid. The principle of the CDCC method is to determine, as accurately as possible, the scattering and dissociation cross sections of a nucleus with a simplified treatment of the final projectile continuum states. To this end, these states describing the relative motions of the unbound fragments are approximately described by square-integrable wave functions at discrete energies. The relative motion between the projectile and target and various cross sections can then be obtained by solving a system of coupled-channel equations. The number of these equations and hence the difficulty of the numerical treatment increase with increasing energy.

The CDCC method was suggested by Rawitscher [45] and first applied to deuteron + nucleus elastic scattering and breakup reactions. It was then extensively developed and used by several groups [17, 46, 47, 48, 49, 50, 51]. Its interest has been revived by the availability of radioactive beams of weakly bound nuclei dissociating into two [18, 47, 48, 49, 50, 51] or three [52, 53, 20, 21] fragments.

We assume that the breakup process leads to $N$ clusters and that the clustertarget interactions do not depend on the target spin. The projectile wave functions $\phi_{\tau}^{J M}\left(E_{\tau B}^{J}, \xi\right)$ describing $N$-body bound states at negative energies $E_{\tau B}^{J}$ and $\phi_{\tau}^{J M}(E, \xi)$ describing $N$-body scattering states at positive energies $E$ are defined with Eq. 2.1). Since the total angular momentum of the projectile-target system is a good quantum number, the first step consists in determining partial waves of the $(N+1)$-body Hamiltonian (2.4). The general partial wave function for a total angular momentum $J_{\mathrm{T}}$ can be expanded over the projectile eigenstates as

$$
\begin{aligned}
\Psi^{J_{\mathrm{T}} M_{\mathrm{T}}}(\mathbf{R}, \xi)= & \sum_{L J \tau} \sum_{B}\left[\phi_{\tau}^{J}\left(E_{\tau B}^{J}, \xi\right) \otimes \psi_{J \tau B}^{L}(\mathbf{R})\right]^{J_{\mathrm{T}} M_{\mathrm{T}}} \\
& +\sum_{L J \tau} \int_{0}^{\infty}\left[\phi_{\tau}^{J}(E, \xi) \otimes \psi_{J \tau E}^{L}(\mathbf{R})\right]^{J_{\mathrm{T}} M_{\mathrm{T}}} d E .
\end{aligned}
$$

In this expansion, index $B$ runs over the bound states of the projectile. The total angular momentum $J_{\mathrm{T}}$ results from the coupling of the orbital momentum $L$ of the projectile-target relative motion with the total angular momentum $J$ of the projectile 
state. The relative-motion partial waves $\psi_{J \tau B}^{L}$ and $\psi_{J \tau E}^{L}$ are unknown and must be determined. The parity is given by the product of $(-1)^{L}$ and the parity of $\phi_{\tau}^{J M}$. The first term of Eq. (5.1) represents the elastic and inelastic channels while the second term is associated with the breakup contribution. However, the presence of the continuum renders this expression intractable.

The basic idea of the CDCC method is to replace wave function 5.1 by

$$
\Psi^{J_{\mathrm{T}} M_{\mathrm{T}}}(\mathbf{R}, \xi)=\sum_{L J \tau n}\left[\phi_{\tau n}^{J}(\xi) \otimes \psi_{J \tau n}^{L}(\mathbf{R})\right]^{J_{\mathrm{T}} M_{\mathrm{T}}},
$$

where the functions $\phi_{\tau n}^{J M}(\xi) \equiv \phi_{\tau}^{J M}\left(E_{\tau n}^{J}, \xi\right)$ represent either bound states $\left(E_{\tau B}^{J}<\right.$ $0)$ or square-integrable approximations of continuum wave functions $\left(E_{\tau n}^{J}>0\right)$ at discrete energies

$$
E_{\tau n}^{J}=\left\langle\phi_{\tau n}^{J M}(\xi)\left|H_{0}\right| \phi_{\tau n}^{J M}(\xi)\right\rangle .
$$

Approximation (5.2) resembles usual coupled-channel expansions and can be treated in a similar way.

In practice, two methods are available to perform the continuum discretization. In the "pseudostate" approach, the Schrödinger equation (2.1) is solved approximately by diagonalizing the projectile Hamiltonian $H_{0}$ either within a finite basis of squareintegrable functions or in a finite region of space. In both cases, square-integrable pseudostates $\phi_{\tau n}^{J M}$ are obtained. This approach is simple but there is little control on the obtained energies $E_{\tau n}^{J}$. Therefore, it is customary to keep only the pseudostates with energies below some limit $E_{\max }$.

The alternative is to separate the integral over $E$ in Eq. 5.1) into a limited number of small intervals, or "bins", $\left[E_{n-1}, E_{n}\right]$ which may depend on $J$ and to use in each of them some average of the exact scattering states in this range of energies [17, 45, 46, 47]. This "bin" method provides the square-integrable basis functions

$$
\phi_{\tau n}^{J M}(\xi)=\frac{1}{W_{n}} \int_{E_{n-1}}^{E_{n}} \phi_{\tau}^{J M}(E, \xi) f_{n}(E) d E
$$

where the weight functions $f_{n}$ may also depend on $J$. Such states are orthogonal because of the orthogonality of the scattering states and they are normed if $W_{n}$ is the norm of $f_{n}$ over $\left[E_{n-1}, E_{n}\right]$. Using Eq. (5.4), their energy (5.3) is given by

$$
E_{\tau n}^{J}=\frac{1}{W_{n}^{2}} \int_{E_{n-1}}^{E_{n}}\left|f_{n}(E)\right|^{2} E d E .
$$

Here also, a maximum energy $E_{\max } \equiv E_{n_{\max }}$ is chosen. In practice, these basis states are usually constructed by averaging the scattering states $\tilde{\phi}_{\tau}^{J M}(k, \xi)$ normalized over the wave number $k$, often within equal momentum intervals [18].

The total wave function (5.2) can be rewritten as

$$
\Psi^{J_{\mathrm{T}} M_{\mathrm{T}}}(\mathbf{R}, \xi)=R^{-1} \sum_{c} \Phi_{c}^{J_{\mathrm{T}} M_{\mathrm{T}}}\left(\Omega_{R}, \xi\right) u_{c}^{J_{\mathrm{T}}}(R),
$$


where $c$ represents the channel $L J \tau n$ and

$$
\Phi_{c}^{J_{\mathrm{T}} M_{\mathrm{T}}}\left(\Omega_{R}, \xi\right)=i^{L}\left[\phi_{\tau n}^{J}(\xi) \otimes Y_{L}\left(\Omega_{R}\right)\right]^{J_{\mathrm{T}} M_{\mathrm{T}}} .
$$

By inserting expansion (5.6) in the Schrödinger equation (2.4) and using Eq. (5.3), the relative wave functions $u_{c}^{J_{\mathrm{T}}}$ are given by a set of coupled equations

$$
\left[-\frac{\hbar^{2}}{2 \mu}\left(\frac{d^{2}}{d R^{2}}-\frac{L(L+1)}{R^{2}}\right)+E_{c}-E_{\mathrm{T}}\right] u_{c}^{J_{\mathrm{T}}}(R)+\sum_{c^{\prime}} V_{c, c^{\prime}}^{J_{\mathrm{T}}}(R) u_{c^{\prime}}^{J_{\mathrm{T}}}(R)=0
$$

where $E_{c} \equiv E_{\tau n}^{J}$. The sum over $L$ is truncated at some value $L_{\max }$. The sum over the pseudo-states or bins is limited by the selected maximum energy $E_{\max }$. The CDCC problem is therefore equivalent to a system of coupled equations where the potentials are given by

$$
V_{c, c^{\prime}}^{J_{\mathrm{T}}}(R)=\left\langle\Phi_{c}^{J_{\mathrm{T}} M_{\mathrm{T}}}\left(\Omega_{R}, \xi\right)\left|V_{P T}(\mathbf{R}, \xi)\right| \Phi_{c^{\prime}}^{J_{\mathrm{T}} M_{\mathrm{T}}}\left(\Omega_{R}, \xi\right)\right\rangle .
$$

This matrix element involves a multidimensional integral over $\Omega_{R}$ and over the internal coordinates $\xi$. In general, the potentials are expanded into multipoles corresponding to the total angular momentum operator $\mathbf{J}_{\mathrm{T}}$ of the system. This may allow an analytical treatment of angular integrals.

System 5.8 must be solved with the boundary condition

$$
u_{c}^{J_{\mathrm{T}}}(R) \underset{R \rightarrow \infty}{\longrightarrow} v_{c}^{-1 / 2}\left[I_{c}\left(K_{c} R\right) \delta_{c c_{0}}-O_{c}\left(K_{c} R\right) S_{c c_{0}}^{J_{\mathrm{T}}}\right],
$$

where $c_{0}$ is the incoming channel. The asymptotic momentum in channel $c$ reads

$$
K_{c}=\sqrt{2 \mu\left(E_{\mathrm{T}}-E_{c}\right) / \hbar^{2}},
$$

and $v_{c}=\hbar K_{c} / \mu$ is the corresponding velocity. In Eq. (5.10), $I_{c}=G_{c}-i F_{c}$ and $O_{c}=$ $I_{c}^{*}$ are the incoming and outgoing Coulomb functions, respectively [29], and the element $S_{c_{c_{0}}}^{J_{\mathrm{T}}}$ of the collision matrix is the amplitude for populating channel $c$ from initial channel $c_{0}$.

Various methods have been developed to solve system (5.8) (see, e.g., Ref. [54]). A convenient approach is the $R$-matrix formalism [55], which is both simple and accurate. The configuration space is divided into two regions: the internal $(R<a)$ and external $(R>a)$ regions, where $a$ is the channel radius. In the external region, the potential matrix defined by Eq. (5.9) can be well approximated by its diagonal Coulomb asymptotic form. Hence the wave function is replaced by combinations of Coulomb functions. In the internal region, the radial wave functions $u_{c}^{J_{\mathrm{T}}}$ can be expanded over some basis [55]. A significant simplification occurs when using Lagrange functions [56, 57, 51].

A scattering wave function verifying the initial condition (2.6) is then constructed with the different partial waves. Inserting this CDCC approximate wave function in Eq. (4.11) enables calculating transition matrix elements towards pseudostates or 


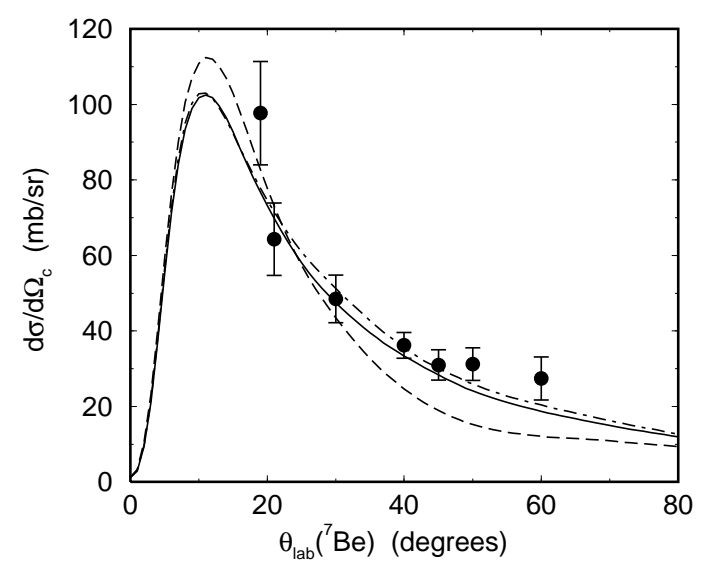

Fig. $4{ }^{7} \mathrm{Be}$ angular distribution after the breakup of ${ }^{8} \mathrm{~B}$ on ${ }^{58} \mathrm{Ni}$ at $25.8 \mathrm{MeV}$ computed within a CDCC model [18]. The convergence of the numerical scheme is illustrated with various maximum values of the ${ }^{7} \mathrm{Be}-\mathrm{p}$ relative orbital momentum $l$ in the continuum and various maximum values of multipole order $\lambda$ of the potential expansion in Eq. [5.9): $l \leq 3, \lambda \leq 2$ (dashed line), $l \leq 3, \lambda \leq 3$ (full line), $l \leq 4, \lambda \leq 4$ (dash-dotted line). Experimental data from Ref. [62]. Reprinted figure with permission from Ref. [18]. Copyright (2001) by the American Physical Society.

bin states as a function of the collision matrices $S^{J_{\mathrm{T}}}$ (see Eq. (5) of Ref. [18]). Since these transition matrix elements are obtained only at discrete energies $E_{n \tau}^{J}$, they must be interpolated in order to obtain breakup cross sections at all energies.

The CDCC method has first been applied to two-body projectiles. As an example, Fig. 44 shows the convergence of the breakup of ${ }^{8} \mathrm{~B}$ on ${ }^{58} \mathrm{Ni}$ at $25.8 \mathrm{MeV}$. The convergence concerns the set of partial waves $l$ in the ${ }^{7} \mathrm{Be}$-p continuum of the projectile and the number of multipoles in the expansion of the potential appearing in matrix elements (5.9). The validity of CDCC has been tested for breakup observables in a comparison with three-body Faddeev calculations [24]. The agreement between both sets of results is good except when the coupling with the transfer channel is important.

Let us also mention extensions beyond the simple two-body model of the projectile by allowing the core to be in an excited state [58, 59]. These references present total cross sections for the breakup on a ${ }^{9} \mathrm{Be}$ target of ${ }^{11} \mathrm{Be}$ into ${ }^{10} \mathrm{Be}+\mathrm{n}$ and of ${ }^{15} \mathrm{C}$ into ${ }^{14} \mathrm{C}+\mathrm{n}$ calculated by including core deformations. This extension of CDCC known as XCDCC leads to very long computational times.

The extension of CDCC to three-body projectiles is more recent [52, 60, 53, 20, 21]. The calculations are still much more time-consuming since the projectile wave functions are much more complicated (see Sec. 7). Consequently, the calculation of the potential matrix elements (5.9) raises important numerical difficulties. At present, converged calculations are mainly restricted to elastic scattering [52, 53, 60]. Most breakup calculations still involve limited bases and/or simplifying assumptions [20, 21] but these limitations can be overcome [61]. 


\section{Breakup reactions of two-body projectiles}

\subsection{Two-cluster model}

Most of the reaction models have been applied assuming a two-cluster structure of the projectile. In this section, we specify the expression of the internal Hamiltonian of the projectile and the set of coordinates usually considered in practical applications. We then illustrate the models presented in Sec. 2] and the approximations explained in Secs. 3 5 with various applications to the study of exotic nuclei and nuclear astrophysics.

We consider here projectiles made up of a single fragment $f$ of mass $m_{f}$ and charge $Z_{f} e$, initially bound to a core $c$ of mass $m_{c}$ and charge $Z_{c} e$. The core and fragment are assumed to have spins $s_{c}$ and $s_{f}$. The internal structure of these clusters and of the target is usually neglected although some structure effects can be simulated by the effective potentials.

Let us now particularize the general formalism 2.1 - 2.3 to the present case. The internal coordinates $\xi$ represent the relative coordinate $\mathbf{r}=\mathbf{r}_{f}-\mathbf{r}_{c}$. The structure of the projectile is described by the two-body internal Hamiltonian

$$
H_{0}=\frac{p^{2}}{2 \mu_{c f}}+V_{c f}(\mathbf{r}),
$$

where $\mu_{c f}=m_{c} m_{f} / m_{P}$ is the reduced mass of the core-fragment pair (with $m_{P}=$ $\left.m_{c}+m_{f}\right), \mathbf{p}$ is the momentum operator of the relative motion and $V_{c f}$ is the potential describing the core-fragment interaction. This potential usually includes a central part and a spin-orbit coupling term in addition to a Coulomb potential. In many cases, the potential is deep enough to contain unphysical bound states below the ground state. These unphysical or forbidden states are useful because they allow the wave function representing the physical ground state to exhibit the number of nodes expected from the Pauli principle, as obtained in microscopic descriptions [63]. Although these forbidden states do not play any role in the core-fragment scattering, they could affect breakup properties. However, as shown in Ref. [64], their presence can be ignored because their effect is negligible.

Let $\mathbf{k}$ be the wave vector describing the asymptotic relative motion between the fragments in the projectile continuum. The corresponding energy is thus $E=$ $\hbar^{2} k^{2} / 2 \mu_{c f}$. Notation $\tau$ in Eq. (2.1) corresponds here to the coupling mode, i.e. to the total spin $S$ of the projectile and the relative orbital momentum $l$. The wave functions defined in Eq. 2.1) read

$$
\phi_{l S}^{J M}(E, \mathbf{r})=r^{-1} i^{l}\left[Y_{l}(\Omega) \otimes \chi_{S}\right]^{J M} u_{l S}^{J}(k, r),
$$

where $\chi_{S}$ is a spinor resulting from the coupling of $s_{c}$ and $s_{f}$. The radial functions $u_{l S}^{J}(k, r)$ are normalized according to $\left\langle u_{l S}^{J}(k, r) \mid u_{l S}^{J}\left(k^{\prime}, r\right)\right\rangle=\delta\left(k-k^{\prime}\right)$. The notation $\hat{k}_{\xi}$ in Eq. (2.2) represents the direction $\Omega_{k}$ of $\mathbf{k}$ and the spin orientations $v_{c}$ and $v_{f}$ of the core and fragment spins $s_{c}$ and $s_{f}$. Relation (2.3) between continuum eigenstates 
of $H_{0}$ becomes

$$
\phi_{\Omega_{k}, v_{c} v_{f}}^{(-)}(E, \mathbf{r})=\frac{1}{k} \sum_{l S J M}\left(s_{c} S_{f} v_{c} v_{f} \mid S v\right)(l S M-v v \mid J M) Y_{l}^{M-v *}\left(\Omega_{k}\right) \phi_{l S}^{J M}(E, \mathbf{r})(6.3)
$$

with the property $\left\langle\phi_{\Omega_{k}, v_{c} v_{f}}^{(-)}(E, \mathbf{r}) \mid \phi_{\Omega_{k}^{\prime}, v_{c}^{\prime} v_{f}^{\prime}}^{(-)}\left(E^{\prime}, \mathbf{r}\right)\right\rangle=\delta\left(\mathbf{k}-\mathbf{k}^{\prime}\right) \delta_{v_{c} v_{c}^{\prime}} \delta_{v_{f} v_{f}^{\prime}}$. Notice that notations $\tau$ and $\hat{k}_{\xi}$ are model dependent and would be quite different if a tensor interaction were included in $V_{c f}$. A detailed description of the simple case $s_{c}=s_{f}=$ 0 can be found in Ref. [26].

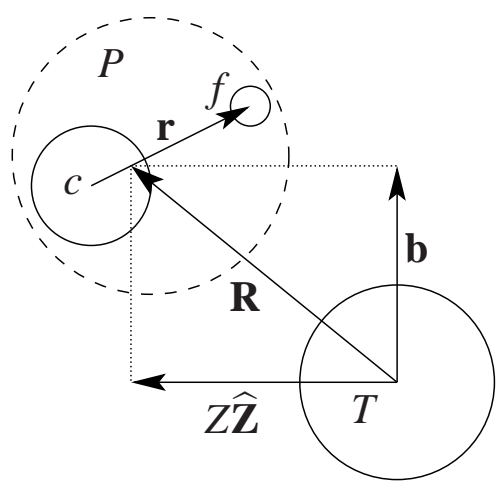

Fig. 5 Jacobi set of coordinates:

$\mathbf{r}$ is the projectile internal coordinate, and

$\mathbf{R}=\mathbf{b}+Z \widehat{\mathbf{Z}}$ is the target-projectile coordinate

Within this framework the description of the reaction reduces to the resolution of a three-body Schrödinger equation (2.4) that reads, in the Jacobi set of coordinates illustrated in Fig. 5 ,

$$
\left[\frac{P^{2}}{2 \mu}+H_{0}+V_{P T}(\mathbf{r}, \mathbf{R})\right] \Psi(\mathbf{r}, \mathbf{R})=E_{\mathrm{T}} \Psi(\mathbf{r}, \mathbf{R}) .
$$

The projectile-target interaction (2.5) then reads

$$
V_{P T}(\mathbf{R}, \mathbf{r})=V_{c T}\left(\mathbf{R}-\frac{m_{f}}{m_{P}} \mathbf{r}\right)+V_{f T}\left(\mathbf{R}+\frac{m_{c}}{m_{P}} \mathbf{r}\right),
$$

where $V_{c T}$ and $V_{f T}$ are optical potentials that simulate the core-target and fragmenttarget interactions, respectively.

For a two-body projectile, the DEA breakup cross section 4.15) becomes Eq. (46) of Ref. [13]. Integration over $\Omega_{k}$ and summation over $v_{c}$ and $v_{f}$ lead to the energy and angular distribution of the fragments in the $P-T$ c.m. rest frame. With the normalization of the positive-energy states given above, it reads [13]

$$
\frac{d \sigma_{\mathrm{bu}}}{d E d \Omega}=\frac{K K^{\prime}}{2 J_{0}+1} \sum_{M_{0}} \sum_{l J M}\left|\int_{0}^{\infty} b d b J_{\left|M-M_{0}\right|}(q b) S_{k l J M}^{\left(M_{0}\right)}(b)\right|^{2},
$$


where $S_{k l J M}^{\left(M_{0}\right)}$ are coefficients of a partial-wave expansion of the breakup amplitude (4.14) (see Eq. (43) of Ref. [13]). Breakup cross sections are mainly expressed as energy distributions $d \sigma_{\mathrm{bu}} / d E$ as a function of the energy of the relative motion between the fragments. They are obtained by integrating (6.6) over $\Omega$. However, most experimental data concern angular distributions or distributions of the core momentum in the laboratory frame. Note that, in addition, theoretical results should be convoluted with the experimental acceptance and resolution. A change of frame for the theoretical results is thus in general not sufficient to allow a fruitful comparison with experiment.

\subsection{Two-body breakup of exotic nuclei}

A first information that one can extract from experiment concerns the separation energy of the halo neutrons. Indeed, the shape of the breakup cross section and, in particular, its maximum are sensitive to this energy as can be shown at first order of perturbation theory with rather simple models based on the asymptotic behaviour of the halo wave function [65]. An example is given by the breakup of ${ }^{19} \mathrm{C}$ on lead at $67 \mathrm{MeV} /$ nucleon. In Fig. 6 a semi-classical calculation with a ${ }^{18} \mathrm{C}+\mathrm{n}$ two-body model shows that the shape of the experimental data is much better reproduced if the binding energy of ${ }^{19} \mathrm{C}$ is raised from the recommended value $0.53 \mathrm{MeV}$ to 0.65 $\mathrm{MeV}$ [34],
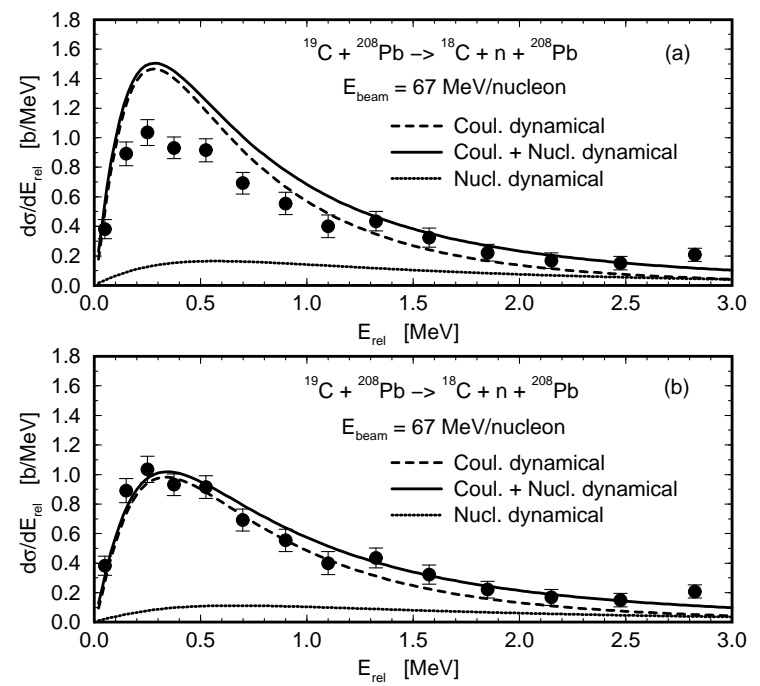

Fig. 6 Breakup of ${ }^{19} \mathrm{C}$ on $\mathrm{Pb}$ at $67 \mathrm{MeV} /$ nucleon: semi-classical cross sections for two different binding energies of the projectile: $0.53 \mathrm{MeV}$ (upper panel) and $0.65 \mathrm{MeV}$ (lower panel) [34]. Experimental data from Ref. [66]. Reprinted figure with permission from Ref. [34]. Copyright (2001) by the American Physical Society. 


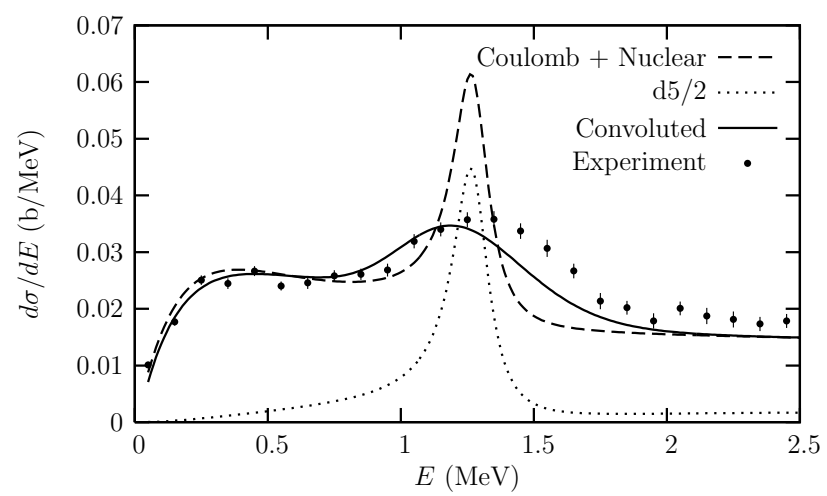

Fig. 7 Breakup of ${ }^{11} \mathrm{Be}$ on a $\mathrm{C}$ target at $67 \mathrm{MeV} /$ nucleon: calculation performed in a semi-classical model [69]. Experimental data from Ref. [35].

Indirect information can also be obtained on the spin of the ground state of the halo nucleus when few rather different orbital momenta are probable. The magnitude of the cross section is very sensitive to the orbital momentum $l$ of the ground state. A study of the one-neutron removal cross section from ${ }^{31} \mathrm{Ne}$ described in a simple ${ }^{30} \mathrm{Ne}+\mathrm{n}$ model allows to rule out the prediction $7 / 2^{-}$of the naive shell model and to confirm the value $3 / 2^{-}$resulting from a shell inversion [67, 68].

Nuclear-induced two-body breakup on light targets is an interesting tool to observe resonances of a halo nucleus and to assess some of their properties. In Fig. 7 are displayed experimental data on the ${ }^{11} \mathrm{Be}$ breakup on a $\mathrm{C}$ target at $67 \mathrm{MeV} /$ nucleon [35]. These data present a broad bump near the location of a known resonance with an assumed spin-parity $5 / 2^{+}$. The bump width is however broader than the known resonance width. A semi-classical calculation (dashed line) based on a ${ }^{10} \mathrm{Be}+\mathrm{n}$ model reproduces the shape of the data very well after convolution with the experimental resolution (full line). Moreover the $d 5 / 2$ component of the theoretical cross section (dotted line) resonates and confirms the $5 / 2^{+}$attribution.

Breakup reactions are also used to infer the spectroscopic factor of the dominant configuration in the core+nucleon structure of halo nuclei [25, 40]. Various theoretical studies have been performed to assess the sensitivity of breakup calculations to the projectile description [70, 71]. These studies have revealed that the breakup cross sections not only depend on the initial bound state of the projectile, but are also sensitive to the description of its continuum [70]. Moreover it has been shown that, for loosely-bound projectiles, only the tail of the wave function is probed in the breakup process and not its whole range [71, 72]. These studies therefore suggest that one should proceed with caution when extracting spectroscopic factors of weakly-bound nuclei from breakup measurements, as other structure properties, like the continuum description, may hinder that extraction.

As mentioned earlier, many Coulomb-breakup experiments have been analyzed within the framework of the first order of the perturbation theory (see Sec. 3.3). In order to assess the validity of that approximation, various authors have com- 


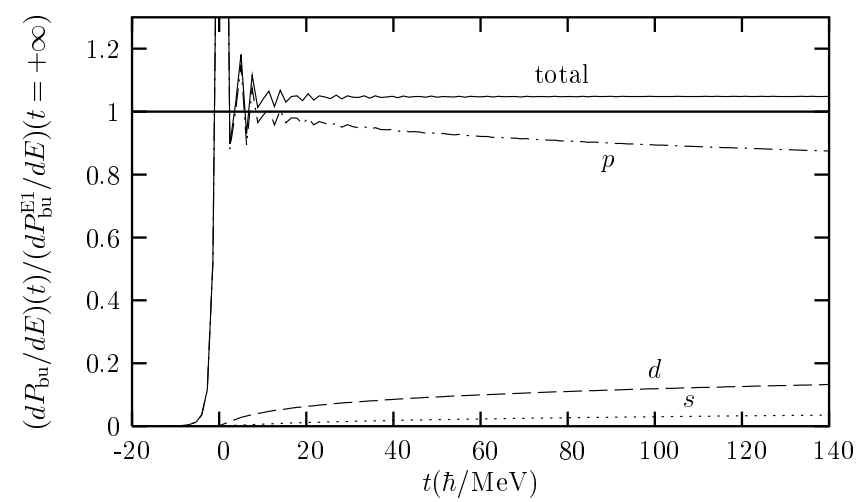

Fig. 8 Influence of the couplings inside the continuum [28]. Time evolution of the numerical breakup probability per energy unit 3.7 for ${ }^{11} \mathrm{Be}$ impinging on $\mathrm{Pb}$ at about $45 \mathrm{MeV} /$ nucleon for a ${ }^{10} \mathrm{Be}-\mathrm{n}$ relative energy $E=1.5 \mathrm{MeV}$ and an impact parameter $b=100 \mathrm{fm}$. Reprinted figure with permission from Ref. [28]. Copyright (2005) by the American Physical Society.

pared perturbation calculations to numerical resolutions of the time-dependent Schrödinger equation [73, 74, 75, 28]. These studies have shown that, in many cases, breakup cannot be modelled as a one-step process from the initial bound state towards the continuum and that higher-order effects should be considered for a reliable description of the reaction. In particular, they indicate that significant couplings are at play inside the continuum. To illustrate this, Fig. 8 displays the time evolution of the breakup probability per energy unit 3.7 for the collision of ${ }^{11} \mathrm{Be}$ on $\mathrm{Pb}$ at about $45 \mathrm{MeV} /$ nucleon computed within the time-dependent ${ }^{10} \mathrm{Be}+\mathrm{n}$ model of Ref. [10]. The obtained value is divided by its evaluation at the first-order of the perturbation theory (3.15) at $t \rightarrow+\infty$. After a sharp increase at the time of closest approach $t=0$, the breakup probability (full line) oscillates and then stabilizes at a value which differs by about 5\% from its first-order estimate. Although the total breakup probability becomes stable, its partial-wave composition still varies: the dominant $p$ wave contribution (dash-dotted line) is depleted towards the $s$ (dotted line) and especially $d$ (dashed line) ones. This signals couplings inside the continuum, which may affect the evaluation of breakup observables [28, 75]. We will see in the next section that it may perturb the analysis of breakup reactions of astrophysical interest [76, 77, 78, 79].

\subsection{Application to nuclear astrophysics}

Radiative-capture reactions are a crucial ingredient in the determination of the reaction rates in nuclear astrophysics. However the difficulty of their measurement and, in some cases, the scatter of the results has raised interest in indirect methods where the time-reversed reaction is simulated by virtual photons in the Coulomb field of 
a heavy nucleus [80, 81]. The radiative-capture cross section can be extracted from breakup cross sections if one assumes that the dissociation is due to E1 virtual photons and occurs in a single step. A typical example is the ${ }^{7} \mathrm{Be}(\mathrm{p}, \gamma)^{8} \mathrm{~B}$ reaction which has been studied with the breakup of ${ }^{8} \mathrm{~B}$ into ${ }^{7} \mathrm{Be}+\mathrm{p}$ on heavy targets at different energies [82, 83, 84, 85, 62, 86, 87, 88].

Though appealing, the breakup method also faces a number of difficulties. First, while many reactions are dominated by an E1 transition, an E2 contribution to the breakup cross section may not be negligible [73]. Second, higher-order effects, i.e. transitions from the initial bound state into the continuum through several steps may not be negligible [73, 74, 75, 28]. Finally, the nuclear interactions between the projectile and the target may interfere with the Coulomb interaction [34, 10]. Therefore elaborate reaction theories must be used to interpret the experimental data.

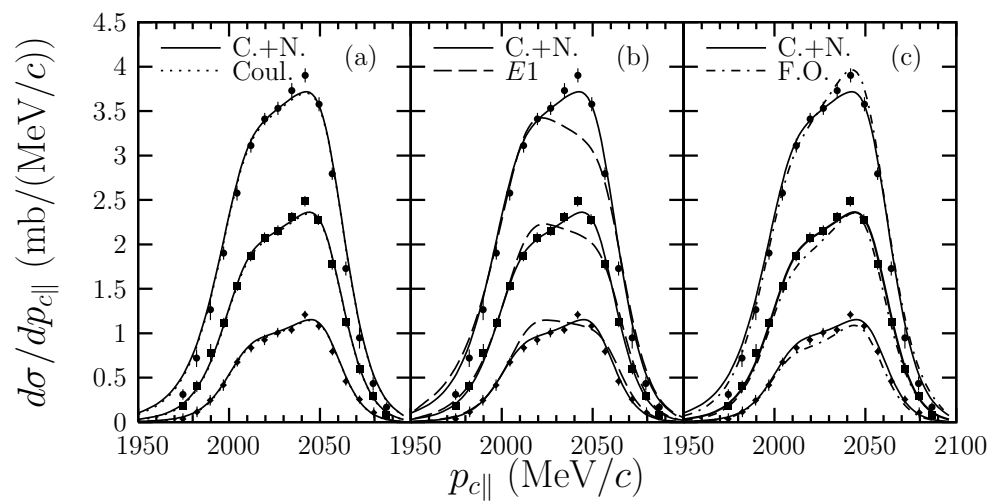

Fig. $9{ }^{8} \mathrm{~B}$ Coulomb breakup on $\mathrm{Pb}$ at $44 \mathrm{MeV} /$ nucleon. Parallel-momentum distribution of the ${ }^{7} \mathrm{Be}$ core corresponding to various angular cuts calculated in a DEA model [77]. (a) Influence of nuclear and Coulomb interactions on the calculation. (b) Effects of the various multipoles of the Coulomb interaction. (c) Role of the higher-order effects on the calculation. Experimental data from Ref. [84].

The experiments on the breakup of ${ }^{8} \mathrm{~B}$ have been analyzed in a number of papers [18, 89, 90, 91, 76, 77]. Figure 9 shows a comparison between the experimental data of Ref. [84] and DEA calculations [77]. Without adjustable parameters, the calculations (full lines) fairly reproduce the asymmetry exhibited by the data which could not be well explained in earlier works [89, 91]. The three panels of Fig. 9 illustrate the influence of various approximations upon the calculation [77]. The left panel illustrates that nuclear $P-T$ interactions can be neglected when data are restricted to forward angles. The central panel confronts a dynamical calculation including only the dipole term of the Coulomb interaction (dashed lines) to the full calculation, indicating that higher multipoles have a significant effect on the breakup process. The right panel compares the dynamical calculation to its first-order approximation (dot-dashed lines), emphasizing the necessity to include higher-order effects 


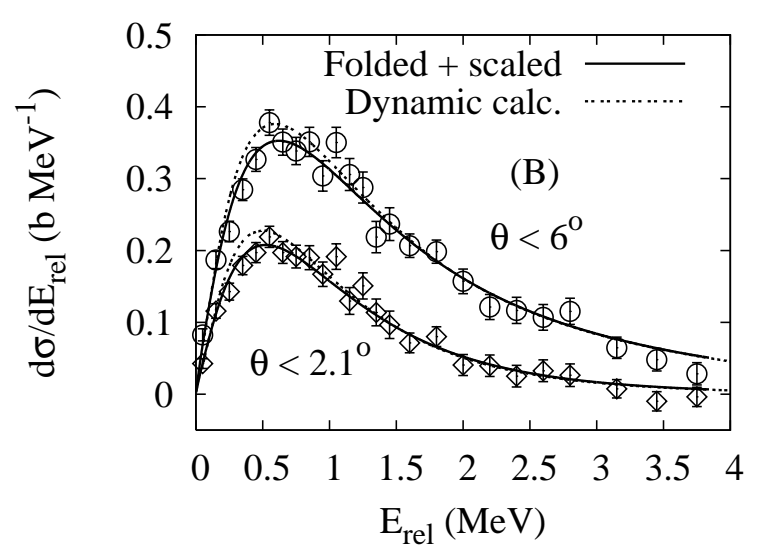

Fig. 10 Breakup of ${ }^{15} \mathrm{C}$ on $\mathrm{Pb}$ at $68 \mathrm{MeV} /$ nucleon. The experimental energy distribution measured for two scattering-angle cuts [98] is confronted to the time-dependent calculation of Ref. [79]. Reprinted figure with permission from Ref. [79]. Copyright (2009) by the American Physical Society.

in breakup calculations. These results show that some of the assumptions of the breakup method [80, 81] are not valid. It is therefore difficult to infer the accuracy of the $S$ factors extracted from breakup cross sections.

An interesting problem was raised by the ${ }^{14} \mathrm{C}(\mathrm{n}, \gamma){ }^{15} \mathrm{C}$ capture reaction. The measured cross sections for the Coulomb breakup of ${ }^{15} \mathrm{C}[92,93$, provided an $S$ factor which disagreed with direct measurements [94, 95]. Moreover, theoretical analyses indicated that the Coulomb-breakup cross sections were inconsistent with information obtained from ${ }^{15} \mathrm{~F}$ by charge symmetry and with microscopic models [96]. A new measurement [97, 98] has obtained breakup cross sections that fully agree with properties of the mirror system and with theory [78, 79]. These theoretical analyses show that a fully dynamical calculation, taking proper account of higher-order effects is necessary to correctly analyze the breakup measurements, in agreement with the analysis of the ${ }^{8} \mathrm{~B}$ Coulomb breakup of Ref. [77]. They also indicate that including both Coulomb and nuclear interactions as well as their interferences is necessary to correctly reproduce data at large scattering angles. In this way a very good agreement can be obtained between direct and indirect measurements of the $S$ factor. Fig.10 displays the breakup cross section of ${ }^{15} \mathrm{C}$ on $\mathrm{Pb}$ measured at $68 \mathrm{MeV} /$ nucleon [98] and its comparison to the theoretical calculation of the time-dependent model of Ref. [79]. The dotted lines show the direct results of the calculation, while the full lines correspond to these results folded by the experimental resolution and scaled to the data. 


\section{Breakup reactions of three-body projectiles}

\subsection{Three-cluster model of projectile}

Let us consider a system of three particles, the core with coordinate $\mathbf{r}_{c}$, mass $m_{c}$ and charge $Z_{c} e$ and two fragments with coordinates $\mathbf{r}_{1}$ and $\mathbf{r}_{2}$, masses $m_{1}$ and $m_{2}$, and charges $Z_{1} e$ and $Z_{2} e$. The projectile mass is $m_{p}=m_{c}+m_{12}$ with $m_{12}=m_{1}+m_{2}$. After removal of the c.m. kinetic energy $T_{\text {c.m. }}$, the three-body Hamiltonian of this system in Eq. 2.1) can be written as

$$
H_{0}=\frac{p_{c}^{2}}{2 m_{c}}+\frac{p_{1}^{2}}{2 m_{1}}+\frac{p_{2}^{2}}{2 m_{2}}+V_{c 1}+V_{c 2}+V_{12}-T_{\text {c.m. }},
$$

where $V_{i j}$ is an effective potential between particles $i$ and $j(i, j=c, 1,2)$. We assume that these interactions involve central, spin-orbit and Coulomb terms. These potentials may contain unphysical bound states below the two-cluster ground state to simulate effects of the Pauli principle. These forbidden states must be eliminated from the three-body wave functions either with pseudopotentials [99] or with supersymmetric transformations [100, 101].

Various resolution techniques can be considered for obtaining the wave functions of a three-body projectile. A first option is to describe this projectile with an expansion in Gaussian functions depending on Jacobi coordinates [102, 30, 20]. For bound states, the wave functions can be obtained from a variational calculation. Well established techniques allow systematic calculations of the matrix elements [102, 30]. Calculations are then simpler when the interactions are expressed in terms of Gaussians. At negative energies, this type of expansion may however have convergence problems in the description of extended halos. At positive energies, it is convenient to obtain pseudostates but not convenient to obtain scattering states.

Let us describe another efficient tool to deal with three-body systems, the formalism of hyperspherical coordinates. It is especially interesting when the two-cluster subsystems are unbound so that only a three-body continuum exists. Notation $\xi$ of Sec. 2. represents here five angular variables and one coordinate with the dimension of a length, the hyperradius (see Refs. [103, 104, 105] for details). Four angular variables correspond to physical angles and the fifth one is related to a ratio of coordinates defined below in Eq. (7.6). The wave functions are expanded in series of hyperspherical harmonics, i.e. a well known complete set of orthonormal functions of the five angular variables. The coefficients are functions of the hyperradius and can be obtained from variational calculations. Scattering states can be obtained from extensions of the $R$ matrix theory [106, 55]. A drawback of this method is that the hyperspherical expansion may converge rather slowly.

With the dimensionless reduced masses $\mu_{c(12)}=m_{c} m_{12} / m_{P} m_{N}$ and $\mu_{12}=m_{1} m_{2}$ $/ m_{12} m_{N}$ where $m_{N}$ is the nucleon mass for example, the internal coordinates $\xi$ are scaled Jacobi coordinates defined as

$$
\mathbf{x}=\sqrt{\mu_{12}}\left(\mathbf{r}_{2}-\mathbf{r}_{1}\right)
$$


and

$$
\mathbf{y}=\sqrt{\mu_{c(12)}}\left(\mathbf{r}_{c}-\frac{m_{1} \mathbf{r}_{1}+m_{2} \mathbf{r}_{2}}{m_{12}}\right),
$$

i.e., up to a scaling factor, the relative coordinate between the clusters 1 and 2 and the relative coordinate of their centre of mass with respect to the core. With Laplacians $\Delta_{x}$ and $\Delta_{y}$ with respect to $\mathbf{x}$ and $\mathbf{y}$, the Hamiltonian (7.1) of this three-body projectile can be rewritten as

$$
H_{0}=-\frac{\hbar^{2}}{2 m_{N}}\left(\Delta_{x}+\Delta_{y}\right)+V_{c 1}+V_{c 2}+V_{12}
$$

To investigate the breakup cross sections for this system, we need wave functions at both positive and negative energies.

In the notation of Refs. [103, 105], the hyperradius $\rho$ and hyperangle $\alpha$ are defined as

$$
\rho=\sqrt{x^{2}+y^{2}}
$$

and

$$
\alpha=\arctan (y / x) .
$$

The hyperangle $\alpha$ and the orientations $\Omega_{x}$ and $\Omega_{y}$ of $\mathbf{x}$ and $\mathbf{y}$ provide a set of five angles collectively denoted as $\Omega_{5}$. The volume element is $d \mathbf{x} d \mathbf{y}=\rho^{5} d \rho d \Omega_{5}$ with $d \Omega_{5}=\sin ^{2} \alpha \cos ^{2} \alpha d \alpha d \Omega_{x} d \Omega_{y}$.

The hyperspherical harmonics form an orthonormal basis which verifies a closure relation. The purely spatial hyperspherical harmonics read [103, 105]

$$
\mathscr{Y}_{K L M_{L}}^{l_{x} l_{y}}\left(\Omega_{5}\right)=\phi_{K}^{l_{x} l_{y}}(\alpha)\left[Y_{l_{x}}\left(\Omega_{x}\right) \otimes Y_{l_{y}}\left(\Omega_{y}\right)\right]^{L M_{L}} .
$$

where $K$ is the hypermomentum quantum number, $l_{x}$ and $l_{y}$ are the orbital quantum numbers associated with $\mathbf{x}$ and $\mathbf{y}$, and $L$ is the quantum number of total orbital momentum. The functions $\phi_{K}^{l_{x} l_{y}}$ depending on the hyperangle $\alpha$ are defined in Eqs. (9) and (10) of Ref. [105]. The hyperspherical harmonics involving spin are defined by

$$
\mathscr{Y}_{\gamma K}^{J M}\left(\Omega_{5}\right)=\left[\mathscr{Y}_{K L}^{l_{x} l_{y}}\left(\Omega_{5}\right) \otimes \chi_{S}\right]^{J M},
$$

where $\chi_{S}$ is a spinor corresponding to a total spin $S$ of the three clusters. Intermediate couplings as, for example, the total spin $s_{12}$ of the fragments are not displayed for simplicity. Index $\gamma$ stands for $\left(l_{x} l_{y} L S\right)$.

A partial wave function $\phi^{J M}$ is a solution of the Schrödinger equation (2.1) associated with the three-body Hamiltonian (7.4) at energy $E$. It can be expanded as

$$
\phi^{J M}\left(E, \rho, \Omega_{5}\right)=\rho^{-5 / 2} \sum_{\gamma K} \chi_{\gamma K}^{J}(E, \rho) \mathscr{Y}_{\gamma K}^{J M}\left(\Omega_{5}\right),
$$


For bound states $(E<0)$, the hyperradial wave functions decrease asymptotically as

$$
\chi_{\gamma K}^{J}(E, \rho) \underset{\rho \rightarrow \infty}{\sim} \exp \left(-\sqrt{2 m_{N}|E| / \hbar^{2}} \rho\right) .
$$

Index $\tau$ of Eq. 2.1) is irrelevant for bound states within the present assumptions. The normalization of the scattering states $(E>0)$ is fixed by their asymptotic form. Several choices are possible. The asymptotic form of the hyperradial scattering wave function is for instance given by [19]

$$
\begin{aligned}
& \chi_{\gamma K\left(\gamma_{\omega} K_{\omega}\right)}^{J}(E, \rho) \underset{\rho \rightarrow \infty}{\rightarrow} i^{K_{\omega}+1}(2 \pi / k)^{5 / 2} \\
& \times\left[H_{K+2}^{-}(k \rho) \delta_{\gamma \gamma_{\omega}} \delta_{K K_{\omega}}-U_{\gamma K, \gamma_{\omega} K_{\omega}}^{J} H_{K+2}^{+}(k \rho)\right],
\end{aligned}
$$

where $k=\sqrt{2 m_{N} E / \hbar^{2}}$ is the wave number and $H_{K}^{-}$and $H_{K}^{+}$are incoming and outgoing functions [107, 108, 106]. In the neutral case, i.e. when clusters 1 and 2 are neutrons, these functions read $H_{K}^{ \pm}(x)= \pm i(\pi x / 2)^{1 / 2}\left[J_{K}(x) \pm i Y_{K}(x)\right]$ where $J_{K}$ and $Y_{K}$ are Bessel functions of first and second kind, respectively. In the charged case, expression (7.11) is only an approximation because the asymptotic form of the Coulomb interaction is not diagonal in hyperspherical coordinates [109, 106]. The indices $\gamma_{\omega} K_{\omega}$ denote the partial entrance channels for this solution. For scattering states, index $\tau$ of Eq. 2.1) is necessary and rather complicated: it represents the entrance channel $\gamma_{\omega} K_{\omega}$. The asymptotic behaviour of a given partial wave depends on the collision matrix. For real interactions, the collision matrix $\mathbf{U}^{J}$ of each partial wave $J$ is unitary and symmetric. For three-body scattering, it differs from two-body collision matrices in an important aspect: its dimension is infinite since the particles can share the angular momentum in an infinity of ways. In practical calculations, its dimension depends on the number of hypermomenta included in the calculation, limited to a maximum $K$ value, denoted as $K_{\max }$.

The three-body final scattering states are described asymptotically with two relative wave vectors. Let $\mathbf{k}_{c}, \mathbf{k}_{1}, \mathbf{k}_{2}$ be the wave vectors of the core and fragments in the projectile rest frame. The asymptotic relative motions are defined by the relative wave vector of the neutrons

$$
\mathbf{k}_{21}=\sqrt{\mu_{12}} \mathbf{k}_{x}=\frac{m_{1} \mathbf{k}_{2}-m_{2} \mathbf{k}_{1}}{m_{12}}
$$

and the relative wave vector of the core with respect to the centre of mass of the fragments

$$
\mathbf{k}_{c(12)}=\sqrt{\mu_{c(12)}} \mathbf{k}_{y}=\frac{m_{12} \mathbf{k}_{c}-m_{c}\left(\mathbf{k}_{1}+\mathbf{k}_{2}\right)}{m_{P}} .
$$

The total internal energy of the projectile with respect to the three-particle threshold is given by 


$$
E=\frac{\hbar^{2}}{2 m_{N}} k^{2}=\frac{\hbar^{2}}{2 m_{N}}\left(k_{x}^{2}+k_{y}^{2}\right) .
$$

The orientations $\Omega_{k_{x}}$ of $\mathbf{k}_{x}$ and $\Omega_{k_{y}}$ of $\mathbf{k}_{y}$ and the ratio $\alpha_{k}=\arctan \left(y_{k} / x_{k}\right)$ form the wave vector hyperangles $\Omega_{5 k}$. The hyperangle $\alpha_{k}$ controls the way the projectile energy $E$ is shared among the fragments. For example, the energy of the relative motion between fragments 1 and 2 is $E \cos ^{2} \alpha_{k}$. In the scattering states (2.2), notation $\hat{k}_{\xi}$ thus represents $\Omega_{5 k}$ and the final orientations $v_{c}, v_{1}, v_{2}$ of the three spins. It is convenient to replace these orientations by the total spin $s_{12}$ of the fragments, the total spin $S$ and its projection $v$. Relation (2.3) is then given by

$$
\begin{aligned}
\phi_{\Omega_{5 k} S v}^{(-)}\left(E, \rho, \Omega_{5}\right)=(2 \pi)^{-3} & \rho^{-5 / 2} \sum_{J M} \sum_{l_{x \omega} l_{y \omega} L_{\omega} K_{\omega}}\left(L_{\omega} S M-v v \mid J M\right) \\
& \times \mathscr{Y}_{l_{x \omega} l_{y \omega} K_{\omega}}^{L}\left(\Omega_{5 k}\right) \phi_{\gamma K\left(\gamma_{\omega} K_{\omega}\right)}^{J M}\left(E, \rho, \Omega_{5}\right) .
\end{aligned}
$$

where $\gamma_{\omega}=\left(l_{x \omega}, l_{y \omega}, L_{\omega}, S\right)$. These functions are normalized with respect to $\delta\left(\mathbf{k}_{\mathbf{x}}-\right.$ $\left.\mathbf{k}_{\mathbf{x}}^{\prime}\right) \boldsymbol{\delta}\left(\mathbf{k}_{\mathbf{y}}-\mathbf{k}_{\mathbf{y}}^{\prime}\right) \delta_{S S^{\prime}} \delta_{v v^{\prime}}$

The hyperradial wave functions $\chi_{\gamma K}^{J}$ are to be determined from the Schrödinger equation 2.1. The parity $\pi=(-1)^{K}$ of the three-body relative motion restricts the sum over $K$ to even or odd values. Rigorously, the summation over $\gamma K$ in (7.9) should contain an infinite number of terms. In practice, this expansion is limited by the truncation value $K_{\max }$. The $l_{x}$ and $l_{y}$ values are limited by $l_{x}+l_{y} \leq K \leq K_{\max }$. For weakly-bound and scattering states, it is well known that the convergence is rather slow and that large $K_{\max }$ values must be used. 106]

The functions $\chi_{\gamma K}^{J}$ are derived from a set of coupled differential equations [105,

$$
\begin{array}{r}
{\left[-\frac{\hbar^{2}}{2 m_{N}}\left(\frac{d^{2}}{d \rho^{2}}-\frac{(K+3 / 2)(K+5 / 2)}{\rho^{2}}\right)-E\right] \chi_{\gamma K}^{J}(E, \rho)} \\
+\sum_{\gamma^{\prime} K^{\prime}} V_{\gamma^{\prime} K^{\prime}, \gamma K}^{J}(\rho) \chi_{\gamma^{\prime} K^{\prime}}^{J}(E, \rho)=0,
\end{array}
$$

where the potentials matrix elements are defined as

$$
V_{\gamma^{\prime} K^{\prime}, \gamma K}^{J}(\rho)=\left\langle\mathscr{Y}_{\gamma^{\prime} K^{\prime}}^{J M}\left(\Omega_{5}\right)\left|\sum_{i>j=1}^{3} V_{i j}\left(\mathbf{r}_{j}-\mathbf{r}_{i}\right)\right| \mathscr{Y}_{\gamma K}^{J M}\left(\Omega_{5}\right)\right\rangle .
$$

For bound states, approximate solutions can be obtained with an expansion on a finite square-integrable basis. However, using such a basis for scattering states raises problems since they do not vanish at infinity. Their asymptotic form requires a proper treatment. This technical difficulty can be solved within the $R$-matrix theory [110, 106, 55] which allows matching a variational function over a finite interval with the correct asymptotic solutions of the Schrödinger equation.

In the $R$-matrix approach, both bound and scattering hyperradial wave functions are approximated over the internal region by an expansion on a set of square- 
integrable variational functions defined over $[0, a]$. Lagrange-mesh basis functions are quite efficient for describing two-body bound and scattering states [56, 111, [12]. The main advantage of this technique is to strongly simplify the calculation of matrix elements (7.17) without loss of accuracy if the Gauss approximation consistent with the mesh is used [105]. This method was extended to three-body bound states in Ref. [105] and to three-body scattering states in Ref. [106]. We refer the reader to those references for details.

\subsection{Dipole strength distribution}

The E1 strength distribution for transitions from the ground state to the continuum is a property of the projectile that can be extracted from breakup experiments under some simplifying assumptions for cases where $\mathrm{E} 1$ is dominant. In the hyperspherical coordinate system, the multipole operators are given by Eq. (B2) of Ref. [105]. For example, in two-neutron halo nuclei, the E1 strength is given by

$$
\mathscr{M}_{\mu}^{\mathrm{E} 1}\left(\rho, \Omega_{5}\right)=e Z_{c} \frac{m_{12}}{m_{P}} \frac{\rho \sin \alpha}{\sqrt{\mu_{c(12)}}} Y_{1}^{\mu}\left(\Omega_{y}\right) .
$$

The E1 transition strength (3.16) from the ground state at negative energy $E_{0}$ with total angular momentum $J_{0}$ to the continuum is given by

$$
\begin{aligned}
\frac{d B(\mathrm{E} 1)}{d E}= & \frac{4}{2 J_{0}+1}\left(\frac{m_{\mathrm{N}}}{\hbar^{2}}\right) E^{2} \sum_{M_{0} \mu} \sum_{S v} \int d \Omega_{5 k} \\
& \left|\left\langle\phi_{\Omega_{5 k} S V}^{(-)}\left(E, \rho, \Omega_{5}\right)\left|\mathscr{M}_{\mu}^{\mathrm{E} 1}\left(\rho, \Omega_{5}\right)\right| \phi^{J_{0} M_{0}}\left(E_{0}, \rho, \Omega_{5}\right)\right\rangle\right|^{2} .
\end{aligned}
$$

The E1 strength presents the advantage that it can also be calculated in various ways without constructing the complicated three-body scattering states [113]. Most model calculations of the E1 strength for ${ }^{6} \mathrm{He}$ indicate a concentration of strength at low energies $E[19,21,107,114,115,116]$. The origin of this low-energy bump remains unclear and can sometimes be attributed to a three-body resonance [107, 19]. The existence of such a bump does not agree with the GSI data [117].

This puzzling problem deserves further studies. A first-order description of Coulomb breakup for ${ }^{6} \mathrm{He}$ is probably not very accurate (see Sec. 7.3), even at the energies of the GSI experiment [117]. Extracting the E1 strength from breakup measurement is very difficult and not without ambiguities. This is exemplified by the variety of experimental results obtained for the breakup of the ${ }^{11} \mathrm{Li}$ two-neutron halo nucleus. As shown in Fig. 11, most early experiments [118, 119, 120] did not display a significant strength at low energies in contradiction with data from the more recent RIKEN experiment [121]. 


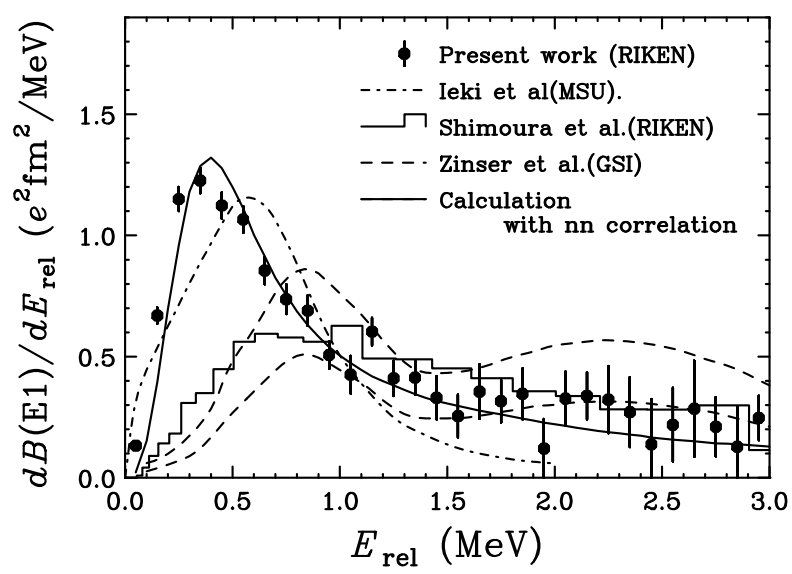

Fig. 11 Experimental E1 strength for the breakup of the ${ }^{11} \mathrm{Li}$ two-neutron halo nucleus: Ref. [121] (full circles), Ref. [118] (dash-dotted line), Ref. [119] (histogram), Ref. [120] (dashed line). Reprinted figure with permission from Ref. [121]. Copyright (2006) by the American Physical Society.

\subsection{The CCE approximation for three-body projectiles}

We consider a collision between a three-body projectile and a structureless target with mass $m_{T}$ and charge $Z_{T} e$ [19]. The breakup reaction is described by the fourbody Schrödinger equation (2.4) where $H_{0}$ is given by Eq. (7.4). The effective potential (2.5) between projectile and target is defined as

$$
\begin{aligned}
V_{P T}(\mathbf{R}, \mathbf{x}, \mathbf{y})= & V_{c T}\left(\mathbf{R}+\frac{m_{12}}{m_{P}} \frac{\mathbf{y}}{\sqrt{\mu_{c(12)}}}\right)+V_{1 T}\left(\mathbf{R}-\frac{m_{c}}{m_{P}} \frac{\mathbf{y}}{\sqrt{\mu_{c(12)}}}-\frac{m_{2}}{m_{12}} \frac{\mathbf{x}}{\sqrt{\mu_{12}}}\right) \\
& +V_{2 T}\left(\mathbf{R}-\frac{m_{c}}{m_{P}} \frac{\mathbf{y}}{\sqrt{\mu_{c(12)}}}+\frac{m_{1}}{m_{12}} \frac{\mathbf{x}}{\sqrt{\mu_{12}}}\right) .
\end{aligned}
$$

In this expression, each interaction $V_{i T}$ between a constituent of the projectile and the target is simulated by a complex optical potential (including a possible Coulomb interaction taking the cluster extension into account).

In order to obtain breakup cross sections, one must calculate transition matrix elements for the breakup into three fragments. The transition matrix elements 4.11) read

$$
\begin{aligned}
T_{f i} & =\left(\mu_{12} \mu_{c(12)}\right)^{-3 / 4} \\
& \times\left\langle e^{i \mathbf{K}^{\prime} \cdot \mathbf{R}} \phi_{\Omega_{5 k} S v}^{(-)}\left(E, \rho, \Omega_{5}\right)\left|V_{P T}(\mathbf{R}, \mathbf{x}, \mathbf{y})\right| \Psi^{\left(M_{0}\right)}\left(\mathbf{R}, \rho, \Omega_{5}\right)\right\rangle
\end{aligned}
$$

for four-body breakup. The factor $\left(\mu_{12} \mu_{c(12)}\right)^{-3 / 4}$ appears when the integration is performed in coordinates $\rho$ and $\Omega_{5}$ and the bound-state wave function $(7.9$ ) is 
normed in this coordinate system rather than in Jacobi coordinates [19]. At the eikonal approximation, the exact scattering wave function $\Psi$ in Eq. 7.21 is replaced by its approximation given by Eqs. (4.1) and (4.17). The transition matrix element $(7.21$ is then obtained following Eq. (4.13) as

$$
T_{f i}=i \hbar v \int d \mathbf{b} e^{-i \mathbf{q} \cdot \mathbf{b}} S_{S v}^{\left(M_{0}\right)}\left(E, \Omega_{5 k}, \mathbf{b}\right)
$$

with the eikonal breakup amplitude (4.14, that reads here [19]

$$
\begin{aligned}
S_{S v}^{\left(M_{0}\right)}\left(E, \Omega_{5 k}, \mathbf{b}\right) & =\left(\mu_{12} \mu_{c(12)}\right)^{-3 / 4} \\
& \times\left\langle\phi_{\Omega_{5 k} S v}^{(-)}\left(E, \rho, \Omega_{5}\right)\left|e^{i \chi\left(\mathbf{b}, \mathbf{s}_{x}, \mathbf{S}_{y}\right)}\right| \phi^{J_{0} M_{0}}\left(E_{0}, \rho, \Omega_{5}\right)\right\rangle .
\end{aligned}
$$

Following Eq. (7.20), the eikonal phase shift $\chi$ defined in Eq. (4.18) is obtained as

$$
\chi=\chi_{c T}+\chi_{1 T}+\chi_{2 T} .
$$

It depends on the transverse part $\mathbf{b}$ of $\mathbf{R}$ as well as on the transverse parts $\mathbf{s}_{x}$ and $\mathbf{s}_{y}$ of the scaled Jacobi coordinates $\mathbf{x}$ and $\mathbf{y}$.

From the transition matrix elements 7.21, various cross sections can be derived. The differential cross section (4.15) with respect to the eight independent variables $\Omega, \mathbf{k}_{21}, \mathbf{k}_{c(12)}$ reads in the c.m. frame

$$
\frac{d \sigma}{d \Omega d \mathbf{k}_{21} d \mathbf{k}_{c(12)}}=\frac{1}{2 J_{0}+1} \frac{1}{4 \pi^{2}}\left(\frac{\mu}{\hbar^{2}}\right)^{2} \frac{K^{\prime}}{K} \sum_{S v M_{0}}\left|T_{f i}\right|^{2} .
$$

The physical wave numbers $k_{21}$ and $k_{c(12)}$ are proportional to $k_{x}$ and $k_{y}$ and can thus be expressed from $k$ and $\alpha_{k}$ [19]. Integrating Eq. (7.25]) over all angles $\Omega$ and $\Omega_{5 k}$ leads to the energy distribution cross section $d \sigma / d E$.

The CCE approximation has allowed calculating various elastic and breakup cross sections for ${ }^{6} \mathrm{He}$ on ${ }^{208} \mathrm{~Pb}$ by treating ${ }^{6} \mathrm{He}$ as an $\alpha+\mathrm{n}+\mathrm{n}$ three-body system [19]. In Fig. 12] the contribution from the different partial waves is displayed at $240 \mathrm{MeV} /$ nucleon. As expected for a transition from a $0^{+}$ground state, the $J=1^{-}$ component is dominant. However the $J=0^{+}$and $J=2^{+}$components are not negligible. The known $2^{+}$resonance at $0.82 \mathrm{MeV}$ is clearly visible in the total cross section. Extracting an E1 strength from such data is thus not easy, even at this high energy.

A comparison of the CCE cross section (full line) with GSI data [117] is presented in Fig.13. The disagreement already discussed for the E1 strength in Sec.7.2 is clearly visible. The data do not show as large a cross section at low energies as the theory. It is not even clear whether the $2^{+}$resonance is visible in these data. Nevertheless the agreement is reasonably good above $2 \mathrm{MeV}$, given that no parameter is fitted to this experiment in the model calculation. The $1^{-}$contribution is calculated with two different ways of eliminating the unphysical bound states in the $\alpha+\mathrm{n}$ potentials (dashed and dotted lines). The low-energy peak corresponds to a 


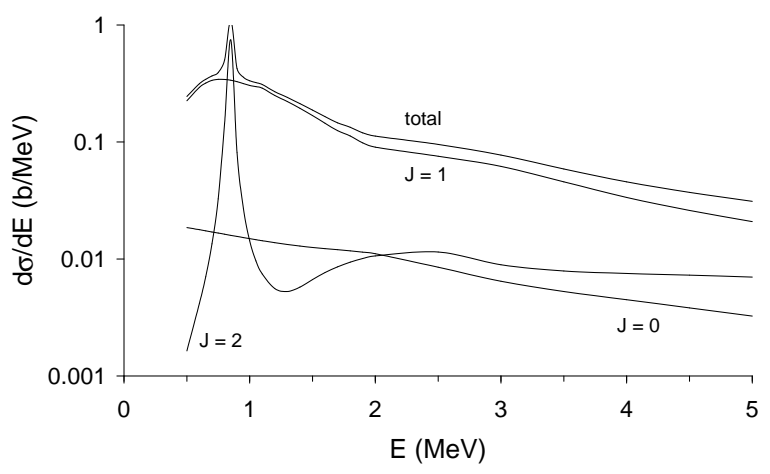

Fig. $12 \mathrm{CCE}$ calculation of the total and $0^{+}, 1^{-}, 2^{+}$partial cross sections of ${ }^{6} \mathrm{He}$ breakup on ${ }^{208} \mathrm{~Pb}$ at $240 \mathrm{MeV} /$ nucleon [19]. Reprinted figure with permission from Ref. [19]. Copyright (2009) by the American Physical Society.

broad resonance in the lowest $1^{-}$three-body phase shift. Further experimental and theoretical works are needed to explain this discrepancy.

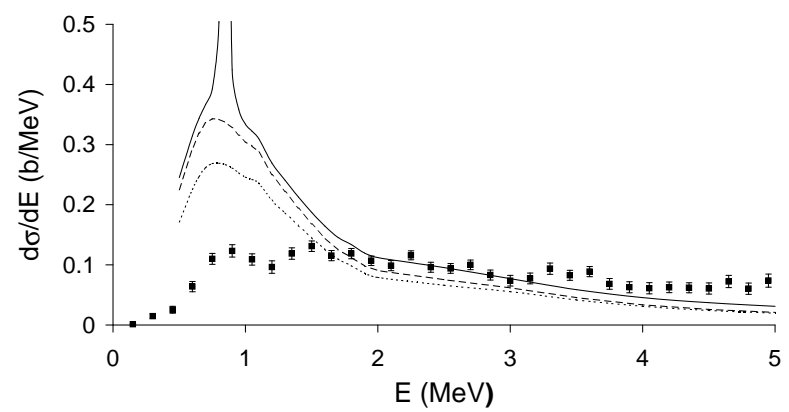

Fig. 13 Comparison [19] between the total CCE cross section (full line) of ${ }^{6} \mathrm{He}$ breakup on ${ }^{208} \mathrm{~Pb}$ at $240 \mathrm{MeV} /$ nucleon with the experimental data of Ref. [117]. The $1^{-}$partial cross sections calculated with two types of elimination of forbidden states (supersymmetry: dashed line, projection: dotted line) are also displayed. Reprinted figure with permission from Ref. [19]. Copyright (2009) by the American Physical Society.

The advantage of the relative simplicity of the CCE is that various types of angular differential cross sections can be calculated. Examples of double differential cross sections showing various energy repartitions between the fragments are presented in Fig. 7 of Ref. [19]. 


\subsection{The CDCC approximation for three-body projectiles}

The CDCC method has also been extended to three-body projectiles. In the first works [52, 60, 53], the pseudostate discretization was adopted. Indeed, it avoids the difficult construction of scattering states and allows an accurate treatment using expansions involving Gaussians with various widths. Only recently was the construction of bins attempted [21]. The difficulty of the calculation restricted the first applications to elastic scattering.

The differential cross section for elastic scattering of ${ }^{6} \mathrm{He}$ on ${ }^{12} \mathrm{C}$ at $229.8 \mathrm{MeV}$ is displayed in Fig. 14 A single-channel calculation neglecting breakup channels (dotted line) overestimates the experimental data of Ref. [122]. The shape of the data is very well reproduced by introducing $0^{+}$and $2^{+}$pseudochannels and taking account of all couplings (full line). In Fig. 15 is displayed a comparison between calculations of ${ }^{6} \mathrm{He}$ elastic scattering on ${ }^{209} \mathrm{Bi}$ at $22.5 \mathrm{MeV}$ involving two-cluster (three-body, dashed line) and three-cluster (four-body, full line) descriptions of ${ }^{6} \mathrm{He}$. A significant difference appears between calculations neglecting breakup channels ("no coupling") and those including it ("full coupling"). The agreement with experimental data [123, 124] seems better within the four-body treatment including the breakup channels.

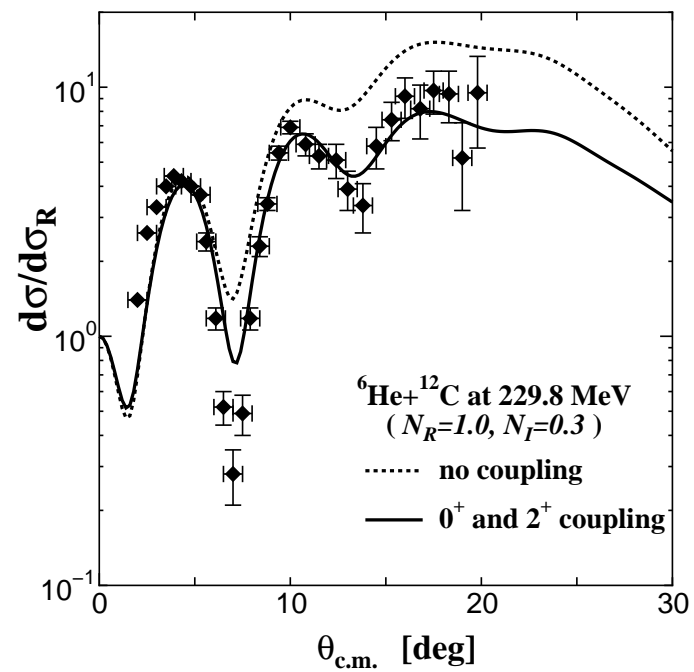

Fig. 14 Ratios of differential cross sections obtained with CDCC to Rutherford cross section for the elastic scattering of ${ }^{6} \mathrm{He}$ on ${ }^{12} \mathrm{C}$ at $229.8 \mathrm{MeV}$ without and with coupling to breakup channels [52]. Experimental data from Ref. [122]. Reprinted figure with permission from Ref. [52]. Copyright (2004) by the American Physical Society.

Another type of basis functions, based on deformed oscillators, has been used to construct ${ }^{6} \mathrm{He}$ pseudostates in Ref. [53]. This technique also allowed a description 


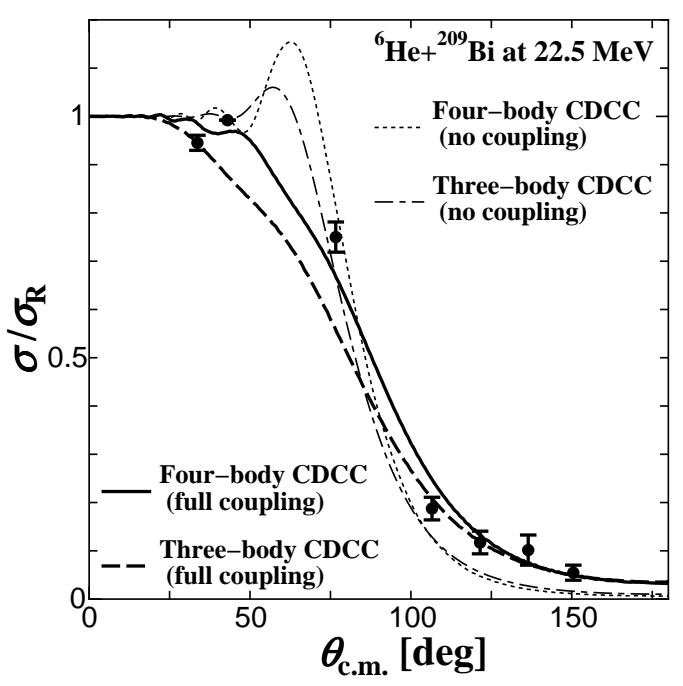

Fig. 15 Ratios of differential cross sections to Rutherford cross section for the elastic scattering of ${ }^{6} \mathrm{He}$ on ${ }^{209} \mathrm{Bi}$ at $22.5 \mathrm{MeV}$ : comparison of three- and four-body CDCC without and with coupling to breakup channels [60]. Experimental data from Refs. [123] 124]. Reprinted figure with permission from Ref. [60]. Copyright (2006) by the American Physical Society.

of elastic scattering explicitly including breakup channels. In Fig. 16, the elastic scattering of ${ }^{6} \mathrm{He}$ on ${ }^{64} \mathrm{Zn}$ at $13.6 \mathrm{MeV}$ is compared with experimental data from Ref. [125]. These results show that including partial waves up to $J=2$ and taking coupling into account (full line) allow a good agreement with data (dots). Here also, the calculation omitting the coupling to the continuum (dashed line) disagrees with the experimental data. The same basis has recently been extended to the construction of bins [21].

While, for three-body projectiles, the effect of breakup channels has been included for some time in studies of elastic scattering, the determination of breakup cross sections is just starting. Some preliminary calculations have been published recently. Some of them are not fully converged [21] or involve simplifying assumptions [20]. A recent CDCC calculation [61] provides a good agreement with experiment [117] for ${ }^{6} \mathrm{He}$ breakup on ${ }^{12} \mathrm{C}$. For ${ }^{6} \mathrm{He}$ breakup on ${ }^{208} \mathrm{~Pb}$, it does not agree well with experiment and is about a factor of two lower than the CCE results of Ref. [19] displayed in Fig.13. The reasons of these discrepancies are not yet understood. Nevertheless, the CDCC method should allow a precise treatment of three-body breakup in a near future. 


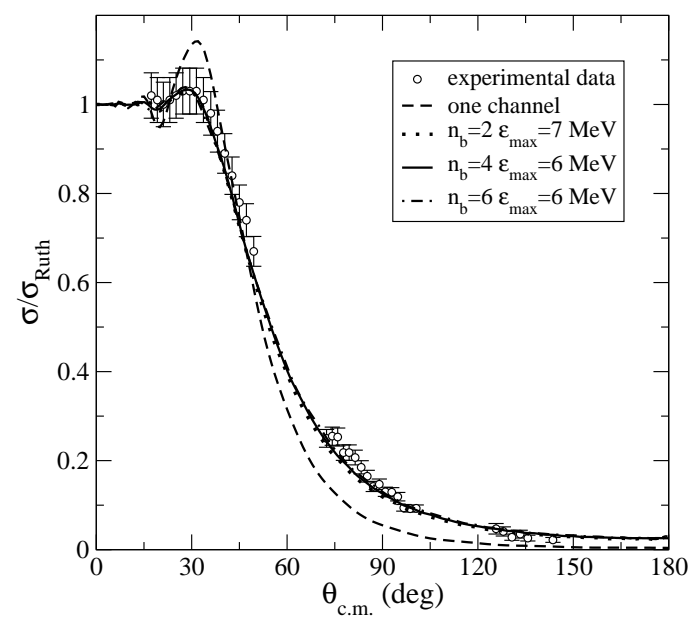

Fig. 16 Ratios of differential cross sections to Rutherford cross section for the elastic scattering of ${ }^{6} \mathrm{He}$ on ${ }^{64} \mathrm{Zn}$ at $13.6 \mathrm{MeV}$ : comparison of CDCC calculations with various basis sizes and maximum energies $E_{\max }$ with a single-channel calculation [53]. Experimental data from Ref. [125]. Reprinted figure with permission from Ref. [53]. Copyright (2008) by the American Physical Society.

\section{Perspectives}

The theory of breakup reactions offers several accurate non-relativistic approximations covering a broad energy range, that allow an interpretation of various experiments. A good accuracy is reached for some time for the breakup of two-body projectiles and is in view for the breakup of three-body projectiles. Good results can already be obtained with the simplest models of projectile structure, provided that the value of the projectile binding energy is correct. This suggests that only limited spectroscopic information can be extracted from the comparison of theory and experiment. This is partly due to the fact that a comparison of experimental data with results of calculations usually requires complicated convolutions. Nevertheless, breakup has proved to be an efficient alternative probe to measure the separation energies of bound states of exotic nuclei. When performed on light targets, it also provides information about the location and width of resonances of such nuclei. Moreover, some information about the quantum numbers of the ground state of exotic nuclei can be assessed from breakup measurements. The extraction of spectroscopic factors, however, is very sensitive to the accuracy of the absolute normalization of experiments. Moreover, the sensitivity of breakup calculations to the description of the continuum of the projectile indicates that these extractions should be performed with caution. In addition, Coulomb breakup on heavy targets is also used to measure astrophysical $S$ factors. However the accuracy of this indirect technique is uncertain. 
Several methods can now be applied to the breakup of three-cluster projectiles (CDCC method, eikonal approximation, ....). They will allow studying coincidence observables that are more difficult to measure but less sensitive to the absolute normalization of cross sections. They should also allow the study of correlations between the emitted fragments. In this respect, efforts should be made at the interface between theory and experiment to facilitate the transformation of the results of model calculations into quantities comparable with the data, taking account of the resolution and acceptances of the detection setup. On the theoretical side, threecluster bound states can be obtained with good accuracy but the difficult treatment of the three-body continuum still requires progress.

Attempts to improve the model description of the projectile by including excited states of the clusters composing the projectile have started with the extended CDCC. In the future, one can expect a further improvement by using a microscopic description of the projectile within the microscopic cluster model [126, 127, 128], involving effective nucleon-nucleon forces and full antisymmetrization. Improvements in the projectile description should first concern bound states. This should reduce the uncertainties appearing in non-microscopic cluster models because of the effective forces between the clusters in the projectile and between the clusters and the target. Using fully antisymmetrized wave functions in breakup calculations seems to be within reach for two-cluster projectiles. This approach should open the way towards ab initio descriptions of the projectile based on fully realistic nucleonnucleon forces.

All the reaction descriptions presented in this review have been developed within non-relativistic quantum mechanics. However, relativistic effects may be significant and affect the analysis of breakup data, even at intermediate energies of a few tens of $\mathrm{MeV} /$ nucleon. Several authors have started analyzing these effects and have proposed ways to take them into account in time-dependent [129] or CDCC [130, 131] frameworks. Since some of the new facilities of radioactive-ion beams will operate at high energies (a few hundreds of MeV/nucleon), these effects will have to be better understood and incorporated in state-of-the-art reaction models.

Acknowledgements We would like to thank P. Descouvemont, G. Goldstein, V. S. Melezhik, F. M. Nunes, and Y. Suzuki for fruitful collaborations concerning the development of some parts of the methods and applications discussed in this text. This text presents research results of BriX (Belgian research initiative on exotic nuclei), the interuniversity attraction pole programme P6/23 initiated by the Belgian-state Federal Services for Scientific, Technical and Cultural Affairs (FSTC). P. C. acknowledges the support of the F.R.S.-FNRS and of the National Science Foundation grant PHY-0800026. The authors also acknowledge travel support of the Fonds de la Recherche Fondamentale Collective (FRFC). 


\section{References}

1. I. Tanihata, H. Hamagaki, O. Hashimoto, S. Nagamiya, Y. Shida, N. Yoshikawa, O. Yamakawa, K. Sugimoto, T. Kobayashi, D.E. Greiner, N. Takahashi, Y. Nojiri, Phys. Lett. B 160, $380(1985)$

2. I. Tanihata, J. Phys. G 22, 157 (1996)

3. B. Jonson, Phys. Rep. 389, 1 (2004)

4. K. Alder, A. Winther, Electromagnetic Excitation (North-Holland, Amsterdam, 1975)

5. T. Kido, K. Yabana, Y. Suzuki, Phys. Rev. C 50, R1276 (1994)

6. H. Esbensen, G.F. Bertsch, C.A. Bertulani, Nucl. Phys. A 581, 107 (1995)

7. S. Typel, H.H. Wolter, Z. Naturforsch. Teil A 54, 63 (1999)

8. V.S. Melezhik, D. Baye, Phys. Rev. C 59, 3232 (1999)

9. D. Lacroix, J.A. Scarpaci, P. Chomaz, Nucl. Phys. A 658, 273 (1999)

10. P. Capel, D. Baye, V.S. Melezhik, Phys. Rev. C 68, 014612 (2003)

11. R.J. Glauber, in Lectures in Theoretical Physics, vol. 1, ed. by W.E. Brittin, L.G. Dunham (Interscience, New York, 1959), p. 315

12. D. Baye, P. Capel, G. Goldstein, Phys. Rev. Lett. 95, 082502 (2005)

13. G. Goldstein, D. Baye, P. Capel, Phys. Rev. C 73, 024602 (2006)

14. J. Margueron, A. Bonaccorso, D.M. Brink, Nucl. Phys. A 720, 337 (2003)

15. B. Abu-Ibrahim, Y. Suzuki, Prog. Theor. Phys. 112, 1013 (2004)

16. P. Capel, D. Baye, Y. Suzuki, Phys. Rev. C 78, 054602 (2008)

17. M. Kamimura, M. Yahiro, Y. Iseri, Y. Sakuragi, H. Kameyama, M. Kawai, Prog. Theor. Phys. Suppl. 89, 1 (1986)

18. J.A. Tostevin, F.M. Nunes, I.J. Thompson, Phys. Rev. C 63, 024617 (2001)

19. D. Baye, P. Capel, P. Descouvemont, Y. Suzuki, Phys. Rev. C 79, 024607 (2009)

20. T. Egami, T. Matsumoto, K. Ogata, M. Yahiro, Prog. Theor. Phys. 121, 789 (2009)

21. M. Rodríguez-Gallardo, J.M. Arias, J. Gómez-Camacho, A.M. Moro, I.J. Thompson, J.A. Tostevin, Phys. Rev. C 80, 051601 (2009)

22. A. Deltuva, A.C. Fonseca, P.U. Sauer, Phys. Rev. C 71, 054005 (2005)

23. A. Deltuva, A.C. Fonseca, P.U. Sauer, Phys. Rev. Lett. 95, 092301 (2005)

24. A. Deltuva, A.M. Moro, E. Cravo, F.M. Nunes, A.C. Fonseca, Phys. Rev. C 76, 064602 (2007)

25. T. Nakamura, S. Shimoura, T. Kobayashi, T. Teranishi, K. Abe, N. Aoi, Y. Doki, M. Fujimaki, N. Inabe, N. Iwasa, et al., Phys. Lett. B 331, 296 (1994)

26. D. Baye, Eur. Phys. J.: Special Topics 156, 93 (2008)

27. H. Esbensen, C.A. Bertulani, Phys. Rev. C 65, 024605 (2002)

28. P. Capel, D. Baye, Phys. Rev. C 71, 044609 (2005)

29. M. Abramowitz, I.A. Stegun, Handbook of Mathematical Functions (Dover, New-York, 1970)

30. Y. Suzuki, R.G. Lovas, K. Yabana, K. Varga, Structure and Reactions of Light Exotic Nuclei (Taylor and Francis, London, 2003)

31. T. Kido, K. Yabana, Y. Suzuki, Phys. Rev. C 53, 2296 (1996)

32. M. Fallot, J.A. Scarpaci, D. Lacroix, P. Chomaz, J. Margueron, Nucl. Phys. A 700, 70 (2002)

33. D. Baye, G. Goldstein, P. Capel, Phys. Lett. A 317, 337 (2003)

34. S. Typel, R. Shyam, Phys. Rev. C 64, 024605 (2001)

35. N. Fukuda, T. Nakamura, N. Aoi, N. Imai, M. Ishihara, T. Kobayashi, H. Iwasaki, T. Kubo, A. Mengoni, M. Notani, et al., Phys. Rev. C 70, 054606 (2004)

36. C.A. Bertulani, P. Danielewicz, Introduction to Nuclear Reactions (Institute of Physics Publishing, Bristol, 2004)

37. N. Austern, Direct Nuclear Reaction Theories (Wiley, New-York, 1970)

38. K. Hencken, G. Bertsch, H. Esbensen, Phys. Rev. C 54, 3043 (1996)

39. B. Abu-Ibrahim, Y. Suzuki, Phys. Rev. C 62, 034608 (2000)

40. P.G. Hansen, J.A. Tostevin, Annu. Rev. Nucl. Part. Sci. 53, 219 (2003) 
41. G. Goldstein, Description de la dissociation de noyaux à halo par l'approximation eikonale dynamique. Ph.D. thesis, Université Libre de Bruxelles, Brussels, Belgium (2007). URL http://theses.ulb.ac.be/ETD-db/collection/available/ULBetd-09262007-111801/

42. R.J. Glauber, G. Matthiae, Nucl. Phys. B 21, 135 (1970)

43. B. Abu-Ibrahim, W. Horiuchi, A. Kohama, Y. Suzuki, Phys. Rev. C 77, 034607 (2008)

44. M. Yahiro, K. Minomo, K. Ogata, M. Kawai, Prog. Theor. Phys. 120, 767 (2008)

45. G.H. Rawitscher, Phys. Rev. C 9, 2210 (1974)

46. N. Austern, Y. Iseri, M. Kamimura, M. Kawai, G. Rawitscher, M. Yahiro, Phys. Rep. 154, 125 (1987)

47. F.M. Nunes, I.J. Thompson, Phys. Rev. C 59, 2652 (1999)

48. A.M. Moro, J.M. Arias, J. Gómez-Camacho, I. Martel, F. Pérez-Bernal, R. Crespo, F. Nunes, Phys. Rev. C 65, 011602 (2001)

49. O.A. Rubtsova, V.I. Kukulin, A.M. Moro, Phys. Rev. C 78, 034603 (2008)

50. A.M. Moro, J.M. Arias, J. Gómez-Camacho, F. Pérez-Bernal, Phys. Rev. C 80, 054605 (2009)

51. T. Druet, D. Baye, P. Descouvemont, J.M. Sparenberg, Nucl. Phys. A 845, 88 (2010)

52. T. Matsumoto, E. Hiyama, K. Ogata, Y. Iseri, M. Kamimura, S. Chiba, M. Yahiro, Phys. Rev. C 70, 061601 (2004)

53. M. Rodríguez-Gallardo, J.M. Arias, J. Gómez-Camacho, R.C. Johnson, A.M. Moro, I.J. Thompson, J.A. Tostevin, Phys. Rev. C 77, 064609 (2008)

54. I.J. Thompson, Comp. Phys. Rep. 7, 167 (1988)

55. P. Descouvemont, D. Baye, Rep. Prog. Phys. 73, 036301 (2010)

56. D. Baye, P.H. Heenen, J. Phys. A 19, 2041 (1986)

57. D. Baye, Phys. Stat. Sol. (b) 243, 1095 (2006)

58. N.C. Summers, F.M. Nunes, I.J. Thompson, Phys. Rev. C 73, 031603 (2006)

59. N.C. Summers, F.M. Nunes, I.J. Thompson, Phys. Rev. C 74, 014606 (2006)

60. T. Matsumoto, T. Egami, K. Ogata, Y. Iseri, M. Kamimura, M. Yahiro, Phys. Rev. C 73, 051602 (2006)

61. T. Matsumoto, K. Katō, M. Yahiro, Phys. Rev. C 82, 051602 (2010)

62. J.J. Kolata, V. Guimarães, D. Peterson, P. Santi, R.H. White-Stevens, S.M. Vincent, F.D. Becchetti, M.Y. Lee, T.W. O’Donnell, D.A. Roberts, J.A. Zimmerman, Phys. Rev. C 63, $024616(2001)$

63. B. Buck, H. Friedrich, C. Wheatley, Nucl. Phys. A 275, 246 (1977)

64. P. Capel, D. Baye, V.S. Melezhik, Phys. Lett. B 552, 145 (2003)

65. S. Typel, G. Baur, Nucl. Phys. A 759, 247 (2005)

66. T. Nakamura, N. Fukuda, T. Kobayashi, N. Aoi, H. Iwasaki, T. Kubo, A. Mengoni, M. Notani, H. Otsu, H. Sakurai, S. Shimoura, T. Teranishi, Y.X. Watanabe, K. Yoneda, M. Ishihara, Phys. Rev. Lett. 83, 1112 (1999)

67. T. Nakamura, N. Kobayashi, Y. Kondo, Y. Satou, N. Aoi, H. Baba, S. Deguchi, N. Fukuda, J. Gibelin, N. Inabe, et al., Phys. Rev. Lett. 103, 262501 (2009)

68. W. Horiuchi, Y. Suzuki, P. Capel, D. Baye, Phys. Rev. C 81, 024606 (2010)

69. P. Capel, G. Goldstein, D. Baye, Phys. Rev. C 70, 064605 (2004)

70. P. Capel, F.M. Nunes, Phys. Rev. C 73, 014615 (2006)

71. P. Capel, F.M. Nunes, Phys. Rev. C 75, 054609 (2007)

72. A. García-Camacho, A. Bonaccorso, D.M. Brink, Nucl. Phys. A 776, 118 (2006)

73. H. Esbensen, G.F. Bertsch, Nucl. Phys. A 600, 37 (1996)

74. S. Typel, G. Baur, Phys. Rev. C 64, 024601 (2001)

75. H. Esbensen, G.F. Bertsch, K.A. Snover, Phys. Rev. Lett. 94, 042502 (2005)

76. K. Ogata, S. Hashimoto, Y. Iseri, M. Kamimura, M. Yahiro, Phys. Rev. C 73, 024605 (2006)

77. G. Goldstein, P. Capel, D. Baye, Phys. Rev. C 76, 024608 (2007)

78. N.C. Summers, F.M. Nunes, Phys. Rev. C 78, 011601 (2008); Erratum ibid. 78, 069908 (2008)

79. H. Esbensen, Phys. Rev. C 80, 024608 (2009); Erratum H. Esbensen, R. Reifarth, Phys. Rev. C 80, 059904 (2009)

80. G. Baur, C.A. Bertulani, H. Rebel, Nucl. Phys. A 458, 188 (1986) 
81. G. Baur, H. Rebel, Annu. Rev. Nucl. Part. Sci 46, 321 (1996)

82. T. Motobayashi, N. Iwasa, Y. Ando, M. Kurokawa, H. Murakami, J. Ruan (Gen), S. Shimoura, S. Shirato, N. Inabe, M. Ishihara, et al., Phys. Rev. Lett. 73, 2680 (1994)

83. T. Kikuchi, T. Motobayashi, N. Iwasa, Y. Ando, M. Kurokawa, S. Moriya, H. Murakami, T. Nishio, J. Ruan (Gen), S. Shirato, et al., Phys. Lett. B 391, 261 (1997)

84. B. Davids, D.W. Anthony, S.M. Austin, D. Bazin, B. Blank, J.A. Caggiano, M. Chartier, H. Esbensen, P. Hui, C.F. Powell, et al., Phys. Rev. Lett. 81, 2209 (1998)

85. V. Guimarães, J.J. Kolata, D. Peterson, P. Santi, R.H. White-Stevens, S.M. Vincent, F.D. Becchetti, M.Y. Lee, T.W. O’Donnell, D.A. Roberts, J.A. Zimmerman, Phys. Rev. Lett. 84, $1862(2000)$

86. B. Davids, D.W. Anthony, T. Aumann, S.M. Austin, T. Baumann, D. Bazin, R.R.C. Clement, C.N. Davids, H. Esbensen, P.A. Lofy, et al., Phys. Rev. Lett. 86, 2750 (2001)

87. B. Davids, S.M. Austin, D. Bazin, H. Esbensen, B.M. Sherrill, I.J. Thompson, J.A. Tostevin, Phys. Rev. C 63, 065806 (2001)

88. F. Schümann, S. Typel, F. Hammache, K. Sümmerer, F. Uhlig, I. Böttcher, D. Cortina, A. Förster, M. Gai, H. Geissel, et al., Phys. Rev. C 73, 015806 (2006)

89. J. Mortimer, I.J. Thompson, J.A. Tostevin, Phys. Rev. C 65, 064619 (2002)

90. B. Davids, S. Typel, Phys. Rev. C 68, 045802 (2003)

91. N.C. Summers, F.M. Nunes, J. Phys. G 31, 1437 (2005)

92. Á. Horváth, J. Weiner, A. Galonsky, F. Deák, Y. Higurashi, K. Ieki, Y. Iwata, Á. Kiss, J.J. Kolata, Z. Seres, et al., Astrophys. J. 570, 926 (2002)

93. U. Datta Pramanik, T. Aumann, K. Boretzky, B.V. Carlson, D. Cortina, T.W. Elze, H. Emling, H. Geissel, A. Grünschloß, M. Hellström, et al., Phys. Lett. B 551, 63 (2003)

94. R. Reifarth, M. Heil, R. Plag, U. Besserer, S. Dababneh, L. Dörr, J. Görres, R.C. Haight, F. Käppeler, A. Mengoni, et al., Nucl. Phys. A 758, 787 (2005)

95. R. Reifarth, M. Heil, C. Forssén, U. Besserer, A. Couture, S. Dababneh, L. Dörr, J. Görres, R.C. Haight, F. Käppeler, et al., Phys. Rev. C 77, 015804 (2008)

96. N.K. Timofeyuk, D. Baye, P. Descouvemont, R. Kamouni, I.J. Thompson, Phys. Rev. Lett. 96, $162501(2006)$

97. T. Nakamura, N. Fukuda, N. Aoi, H. Iwasaki, T. Kobayashi, T. Kubo, A. Mengoni, M. Notani, H. Otsu, H. Sakurai, et al., Nucl. Phys. A 722, C301 (2003)

98. T. Nakamura, N. Fukuda, N. Aoi, N. Imai, M. Ishihara, H. Iwasaki, T. Kobayashi, T. Kubo, A. Mengoni, T. Motobayashi, et al., Phys. Rev. C 79, 035805 (2009)

99. V.I. Kukulin, V.N. Pomerantsev, Ann. Phys. 111, 330 (1978)

100. D. Baye, Phys. Rev. Lett. 58, 2738 (1987)

101. D. Baye, J. Phys. A 20, 5529 (1987)

102. E. Hiyama, Y. Kino, M. Kamimura, Prog. Part. Nucl. Phys. 51, 223 (2003)

103. J. Raynal, J. Revai, Nuovo Cim. A 39, 612 (1970)

104. M.V. Zhukov, B.V. Danilin, D.V. Fedorov, J.M. Bang, I.J. Thompson, J.S. Vaagen, Phys. Rep. 231, 151 (1993)

105. P. Descouvemont, C. Daniel, D. Baye, Phys. Rev. C 67, 044309 (2003)

106. P. Descouvemont, E. Tursunov, D. Baye, Nucl. Phys. A 765, 370 (2006)

107. B.V. Danilin, I.J. Thompson, J.S. Vaagen, M.V. Zhukov, Nucl. Phys. A 632, 383 (1998)

108. I.J. Thompson, B.V. Danilin, V.D. Efros, J.S. Vaagen, J.M. Bang, M.V. Zhukov, Phys. Rev. C 61, 024318 (2000)

109. V. Vasilevsky, A.V. Nesterov, F. Arickx, J. Broeckhove, Phys. Rev. C 63, 034607 (2001)

110. A.M. Lane, R.G. Thomas, Rev. Mod. Phys. 30, 257 (1958)

111. M. Hesse, J.M. Sparenberg, F.V. Raemdonck, D. Baye, Nucl. Phys. A 640, 37 (1998)

112. M. Hesse, J. Roland, D. Baye, Nucl. Phys. A 709, 184 (2002)

113. Y. Suzuki, W. Horiuchi, D. Baye, Prog. Theor. Phys. 123, 547 (2010)

114. A. Cobis, D.V. Fedorov, A.S. Jensen, Phys. Rev. Lett. 79, 2411 (1997)

115. T. Myo, K. Katō, S. Aoyama, K. Ikeda, Phys. Rev. C 63, 054313 (2001)

116. K. Hagino, H. Sagawa, Phys. Rev. C 76, 047302 (2007)

117. T. Aumann, D. Aleksandrov, L. Axelsson, T. Baumann, M.J.G. Borge, L.V. Chulkov, J. Cub, W. Dostal, B. Eberlein, T.W. Elze, et al., Phys. Rev. C 59, 1252 (1999) 
118. K. Ieki, D. Sackett, A. Galonsky, C.A. Bertulani, J.J. Kruse, W.G. Lynch, D.J. Morrissey, N.A. Orr, H. Schulz, B.M. Sherrill, et al., Phys. Rev. Lett. 70, 730 (1993)

119. S. Shimoura, T. Nakamura, M. Ishihara, N. Inabe, T. Kobayashi, T. Kubo, R.H. Siemssen, I. Tanihata, Y. Watanabe, Phys. Lett. B 348, 29 (1995)

120. M. Zinser, F. Humbert, T. Nilsson, W. Schwab, H. Simon, T. Aumann, M.J.G. Borge, L.V. Chulkov, J. Cub, T.W. Elze, et al., Nucl. Phys. A 619, 151 (1997)

121. T. Nakamura, A.M. Vinodkumar, T. Sugimoto, N. Aoi, H. Baba, D. Bazin, N. Fukuda, T. Gomi, H. Hasegawa, N. Imai, et al., Phys. Rev. Lett. 96, 252502 (2006)

122. V. Lapoux, N. Alamanos, F. Auger, V. Fékou-Youmbi, A. Gillibert, F. Marie, S. OttiniHustache, J.L. Sida, D.T. Khoa, Y. Blumenfeld, et al., Phys. Rev. C 66, 034608 (2002)

123. E.F. Aguilera, J.J. Kolata, F.M. Nunes, F.D. Becchetti, P.A. DeYoung, M. Goupell, V. Guimarães, B. Hughey, M.Y. Lee, D. Lizcano, et al., Phys. Rev. Lett. 84, 5058 (2000)

124. E.F. Aguilera, J.J. Kolata, F.D. Becchetti, P.A. DeYoung, J.D. Hinnefeld, Á. Horváth, L.O. Lamm, H.Y. Lee, D. Lizcano, E. Martinez-Quiroz, et al., Phys. Rev. C 63, 061603 (2001)

125. A. Di Pietro, P. Figuera, F. Amorini, C. Angulo, G. Cardella, S. Cherubini, T. Davinson, D. Leanza, J. Lu, H. Mahmud, et al., Phys. Rev. C 69, 044613 (2004)

126. K. Wildermuth, Y.C. Tang, A Unified Theory of the Nucleus (Vieweg, Braunschweig, 1977)

127. Y.C. Tang, in Topics in Nuclear Physics II, Lecture Notes in Physics, Springer, Berlin, 145, $571(1981)$

128. P. Descouvemont, M. Dufour, in Cluster in Nuclei - Vol. 2, Lecture Notes in Physics, Springer, Berlin (2010)

129. H. Esbensen, Phys. Rev. C 78, 024608 (2008)

130. C.A. Bertulani, Phys. Rev. Lett. 94, 072701 (2005)

131. K. Ogata, C.A. Bertulani, Prog. Theor. Phys. 121, 1399 (2009) 